\title{
Gravitational waves from black hole-neutron star binaries I: Classification of waveforms
}

\author{
${ }^{1}$ Masaru Shibata, ${ }^{2}$ Koutarou Kyutoku, ${ }^{3}$ Tetsuro Yamamoto, ${ }^{4}$ Keisuke Taniguchi \\ ${ }^{1}$ Yukawa Institute of Theoretical Physics, Kyoto University, Kyoto, 606-8502, Japan \\ ${ }^{2}$ Department of Physics, University of Tokyo, Tokyo 113-0033, Japan \\ ${ }^{3}$ Yugen Club, Toyama, Shinjuku, Tokyo 162-0052, Japan \\ ${ }^{4}$ Department of Physics, University of Wisconsin-Milwaukee, P.O. Box 413, Milwaukee, Wisconsin 53201
}

\begin{abstract}
Using our new numerical-relativity code SACRA, long-term simulations for inspiral and merger of black hole (BH)-neutron star (NS) binaries are performed, focusing particularly on gravitational waveforms. As the initial conditions, BH-NS binaries in a quasiequilibrium state are prepared in a modified version of the moving-puncture approach. The $\mathrm{BH}$ is modeled by a nonspinning moving puncture and for the NS, a polytropic equation of state with $\Gamma=2$ and the irrotational velocity field are employed. The mass ratio of the $\mathrm{BH}$ to the NS, $Q=M_{\mathrm{BH}} / M_{\mathrm{NS}}$, is chosen in the range between 1.5 and 5 . The compactness of the NS, defined by $\mathcal{C}=G M_{\mathrm{NS}} / c^{2} R_{\mathrm{NS}}$, is chosen to be between 0.145 and 0.178 . For a large value of $Q$ for which the NS is not tidally disrupted and is simply swallowed by the $\mathrm{BH}$, gravitational waves are characterized by inspiral, merger, and ringdown waveforms. In this case, the waveforms are qualitatively the same as that from BH-BH binaries. For a sufficiently small value of $Q \lesssim 2$, the NS may be tidally disrupted before it is swallowed by the BH. In this case, the amplitude of the merger and ringdown waveforms is very low, and thus, gravitational waves are characterized by the inspiral waveform and subsequent quick damping. The difference in the merger and ringdown waveforms is clearly reflected in the spectrum shape and in the "cut-off" frequency above which the spectrum amplitude steeply decreases. When an NS is not tidally disrupted (e.g., for $Q=5$ ), kick velocity, induced by asymmetric gravitational wave emission, agrees approximately with that derived for the merger of $\mathrm{BH}-\mathrm{BH}$ binaries, whereas for the case that the tidal disruption occurs, the kick velocity is significantly suppressed.
\end{abstract}

PACS numbers: 04.25.D-, 04.30.-w, 04.40.Dg

\section{INTRODUCTION}

The coalescence of black hole (BH)-neutron star (NS) binaries is one of the most promising sources for kilometer-size laserinterferometric gravitational-wave detectors such as LIGO [1] and VIRGO [2]. A statistical study based on the stellar evolution synthesis suggests that the detection rate of gravitational waves from $\mathrm{BH}$ NS binaries will be about $1 / 20-1 / 3$ of the rate expected for the merger of the NS-NS binaries in the universe [3, 4$]$. Then, the detection rate of such systems will be $\sim 0.5-50$ events per year for advanced detectors such as advancedLIGO [5], and hence, the detection is expected to be achieved in the near future. For clarifying the nature of the sources of gravitational waves and for extracting their physical information, theoretical templates of gravitational waves are necessary for the data analysis. For theoretically computing gravitational waveforms for the late inspiral and merger phases of BH-NS binaries, numerical relativity is the unique approach.

The final fate of coalescing BH-NS binaries is divided into two cases depending primarily on the $\mathrm{BH}$ mass. When the BH mass is small enough, the companion NS will be tidally disrupted before it is swallowed by the $\mathrm{BH}$. By contrast, when the BH mass is large enough, the NS will be swallowed by the $\mathrm{BH}$ without tidal disruption. The latest study for the BH-NS binary in a quasiequilibrium indicates that the tidal disruption of the NSs by a nonspinning $\mathrm{BH}$ will occur for the case that the $\mathrm{BH}$ mass is $\lesssim 4 M_{\odot}$, for the hypothetical mass and radius of the NS, $M_{\mathrm{NS}} \sim 1.35 M_{\odot}$ and $R_{\mathrm{NS}} \sim 11-12 \mathrm{~km}$, respectively [6 8]. The tidally disrupted NSs may form a disk or a torus around the $\mathrm{BH}$ if the tidal disruption occurs outside the innermost stable circular orbit (ISCO). A system consisting of a rotating $\mathrm{BH}$ surrounded by a massive, hot torus has been proposed as one of the likely sources for the central engine of gamma-ray bursts with a short duration [9] (hereafter SGRBs). Hence, the merger of a low-mass $\mathrm{BH}$ and its companion NS can be a candidate of the central engine. According to the observational results by the Swift and HETE-2 satellites [10], the total energy of the SGRBs is larger than $\sim 10^{48} \mathrm{ergs}$, and typically $10^{49}-10^{50}$ ergs. The studies of hypercritical, neutrino-dominated accretion disks around a $\mathrm{BH}$ suggest that disk mass should be larger than $\sim 0.01 M_{\odot}$ for providing such high energy for generating gamma-ray via neutrino process [11, 12]. The question is whether or not the mass and thermal energy of the disk (torus) are large enough for driving the SGRBs of the huge total energy. Numerical-relativity simulation plays an important role for answering this question.

In the last three years, general relativistic numerical simulations for the inspiral and merger of BH-NS binaries have been performed by three groups 13 17]. However, most of these previous works should be regarded as preliminary ones because the simulations were performed only for a short time scale: In the early works [13 15], the inspiral motion is followed for at most two orbits. 
With such short-term simulations, the orbit is not guaranteed to be quasicircular at the onset of the merger, and hence, the obtained results are not likely to be very realistic because of the presence of the unrealistic eccentricity and incorrect approaching velocity. Moreover, by such incorrect conditions at the onset of the merger, the final outcome such as mass and spin of the $\mathrm{BH}$ and the physical condition for the disk surrounding the $\mathrm{BH}$ is not likely to be computed correctly, although a rough qualitative feature of the merger mechanism and gravitational waveforms have been found from these works. In the latest works [16, 17], the inspiral motion is followed for $\sim 4$ orbits, but in each of these works, the simulation was performed only for one model. More systematic study is obviously necessary to clarify the quantitative details about the merger process, the final outcome, and gravitational waveforms.

In this paper, we report our latest work in which a long-term simulation of BH-NS binaries is performed for a wide variety of $\mathrm{BH}$ masses, NS masses, and NS radii for the first time. In the present simulation, the inspiral motion is followed for $4-7$ orbits. With this setting, the eccentricity of the last inspiral motion appears to be negligible and the approaching velocity at the onset of the merger is correctly taken into account. Furthermore, we systematically choose the BH and NS masses and NS radii for a wide range, and as a result, it becomes possible to clarify the dependence of the merger process, final outcome, and gravitational waveforms on the mass ratio of the binary and the compactness of the NS. One drawback in the present work is that the NSs are modeled by a simple equation of state (EOS). However, the various features found in this paper will qualitatively hold irrespective of the EOS and thus the present work will be an important first step toward more detailed simulation in the near future in which the NSs are modeled by more realistic EOSs.

The paper is organized as follows. Section II summarizes the initial conditions chosen in this paper. Section III briefly describes the formulation and methods for the numerical simulation. Section IV presents the numerical results of the simulation focusing primarily on the dependence of the merger mechanism and gravitational waveforms on the mass ratio and radius of NSs. Section $\mathrm{V}$ is devoted to a summary. Throughout this paper, the geometrical units of $c=G=1$, where $c$ and $G$ are the speed of light and gravitational constant, are used, otherwise stated. The irreducible mass of a $\mathrm{BH}$, rest mass of an NS, gravitational mass and circumferential radius of an NS in isolation, Arnowitt-Deser-Misner (ADM) mass of system, and sum of the BH and NS mass (often referred to as the total mass) are denoted by $M_{\mathrm{BH}}, M_{*}$, $M_{\mathrm{NS}}, R_{\mathrm{NS}}, M$, and $m_{0}\left(=M_{\mathrm{BH}}+M_{\mathrm{NS}}\right)$, respectively. The mass ratio of the binary and the compactness of the NS are defined by $Q \equiv M_{\mathrm{BH}} / M_{\mathrm{NS}}$ and $\mathcal{C} \equiv G M_{\mathrm{NS}} / c^{2} R_{\mathrm{NS}}$, respectively. Note that $M_{\mathrm{BH}}$ is equal to the ADM mass of the $\mathrm{BH}$ in isolation for a nonspinning $\mathrm{BH}$. Greek indices and Latin indices denote the spacetime and space components, respectively; Cartesian coordinates are used for the spatial coordinates.

\section{INITIAL CONDITION}

BH-NS binaries in a quasiequilibrium state are employed as an initial condition of the numerical simulation. Following our previous papers [13, 14], the quasiequilibrium state is computed in the moving-puncture framework [18, 19]. The formulation in this framework is slightly different from that in the excision framework which is adopted in most of the previous works [68, 20, 21], although both formulations are based on the conformal-flatness formalism for the three-metric [22]. In the present work, we adopt the same basic equations as those in Ref. 13]. However, we change the condition for defining the center of mass of system to improve the quality of the quasiequilibrium (specifically, to reduce the orbital eccentricity). To clarify which part is changed, we summarize the basic equations and method for determining the quasiequilibrium state again in the following. Detailed numerical solutions and their properties are presented in an accompanied paper [23], to which the reader may refer for details.

\section{A. Formulation}

If the orbital separation of a BH-NS binary is large enough, the time scale of gravitational-wave emission $\left(\tau_{\mathrm{GW}}\right)$ is much longer than the orbital period $\left(P_{0}\right)$. In the present work, we follow the inspiral motion of the BH-NS binaries for more than 4 orbits (typically $\gtrsim 5$ orbits). This implies that the initial binary separation is always large enough to satisfy the condition, $\tau_{\mathrm{GW}} \gg P_{0}$. Thus, the binaries initially given should be in a quasicircular orbit (i.e., the $\mathrm{BH}$ and NS are approximately in an equilibrium in the comoving frame with an angular velocity $\Omega$ ). To obtain such a state, we may assume the presence of a helical Killing vector around the center of mass of the system as follows,

$$
\ell^{\mu}=\left(\partial_{t}\right)^{\mu}+\Omega\left(\partial_{\varphi}\right)^{\mu}
$$

where $\Omega$ denotes the orbital angular velocity.

We assume that the NSs have the irrotational velocity field because it is believed to be realistic for the BH-NS binaries in close orbits [24]. For the fluid of the irrotational velocity field, the Euler and continuity equations reduce to a first integral of motion and an elliptic-type equation for the velocity potential in the presence of the helical Killing vector [25]. As a result, the density profile and velocity field are determined by solving these hydrostatic equations.

For computing a solution of the geometric variables of a quasiequilibrium state, we employ the so-called conformal-flatness formalism for the three-geometry [22]. 
In this formalism, a solution is obtained by solving Hamiltonian and momentum constraint equations, and an equation for the lapse function $(\alpha)$ which is derived by imposing the maximal slicing condition as $K=0=\partial_{t} K$ where $K$ is the trace part of the extrinsic curvature $K_{i j}$. These equations lead to the equations for the conformal factor $\psi$, a rescaled tracefree extrinsic curvature $\hat{A}_{i}{ }^{j} \equiv \psi^{6} K_{i}{ }^{j}$, and a weighted lapse $\Phi \equiv \alpha \psi$ as

$$
\begin{aligned}
& \Delta \psi=-2 \pi \rho_{\mathrm{H}} \psi^{5}-\frac{1}{8} \hat{A}_{i}{ }^{j} \hat{A}_{j}{ }^{i} \psi^{-7}, \\
& \hat{A}_{i, j}{ }^{j}=8 \pi J_{i} \psi^{6} \\
& \Delta \Phi=2 \pi \Phi\left[\psi^{4}\left(\rho_{\mathrm{H}}+2 S\right)+\frac{7}{16 \pi} \psi^{-8} \hat{A}_{i}{ }^{j} \hat{A}_{j}{ }^{i}\right],
\end{aligned}
$$

where $\Delta$ denotes the flat Laplacian, $\rho_{\mathrm{H}}=\rho h\left(\alpha u^{t}\right)^{2}-P$, $J_{i}=\rho h \alpha u^{t} u_{i}$, and $S=\rho h\left[\left(\alpha u^{t}\right)^{2}-1\right]+3 P . \quad \rho$ is the rest-mass density, $h$ is the specific enthalpy defined by $1+\varepsilon+P / \rho, \varepsilon$ is the specific internal energy, $P$ is the pressure, and $u^{\mu}$ is the four-velocity.

For the relation among $\rho, \varepsilon$, and $P$, we adopt the polytropic EOS as,

$$
P=\kappa \rho^{\Gamma},
$$

where $\kappa$ is the adiabatic constant and $\Gamma$ the adiabatic index for which we choose 2 in this paper. In this EOS, $\varepsilon$ is given by $P /(\Gamma-1) \rho$.

We solve the elliptic equations (22)-(41) in the movingpuncture framework [18, 19, 26]. Assuming that the puncture is located at $\boldsymbol{r}_{\mathrm{P}}\left(=x_{\mathrm{P}}^{k}\right)$, we set $\psi$ and $\Phi$ as

$$
\psi=1+\frac{M_{\mathrm{P}}}{2 r_{\mathrm{BH}}}+\phi \text { and } \Phi=1-\frac{M_{\Phi}}{r_{\mathrm{BH}}}+\eta,
$$

where $M_{\mathrm{P}}$ and $M_{\Phi}$ are positive constants of mass dimension, and $r_{\mathrm{BH}}=\left|x_{\mathrm{BH}}^{k}\right|\left(x_{\mathrm{BH}}^{k}=x^{k}-x_{\mathrm{P}}^{k}\right)$. Then, substituting Eq. (6) into Eqs. (2) and (4), elliptic equations for new non-singular functions $\phi$ and $\eta$ are derived.

The mass parameter $M_{\mathrm{P}}$ may be arbitrarily given, and thus, it is appropriately chosen to obtain a desired $\mathrm{BH}$ mass. For a given value of $M_{\mathrm{P}}, M_{\Phi}$ is determined by the virial relation, which should hold for stationary spacetimes (e.g., Ref. [27]), as

$$
\oint_{r \rightarrow \infty} \partial_{i} \Phi d S^{i}=-\oint_{r \rightarrow \infty} \partial_{i} \psi d S^{i}=2 \pi M_{0},
$$

where $M_{0}$ is the initial ADM mass of the system.

For a solution obtained in this formalism, $\alpha$ always becomes negative near the puncture (approximately, inside apparent horizon), but such lapse is not favorable for numerical simulation. Following previous papers [13, 14], thus, the initial condition for $\alpha$ is appropriately modified so as to satisfy the condition of $\alpha>0$ everywhere.

Equation (3) is rewritten by setting

$$
\hat{A}_{i j}\left(=\hat{A}_{i}^{k} \delta_{j k}\right)=W_{i, j}+W_{j, i}-\frac{2}{3} \delta_{i j} \delta^{k l} W_{k, l}+K_{i j}^{\mathrm{P}},
$$

where $W_{i}\left(=W^{i}\right)$ denotes an auxilary three-dimensional function and $K_{i j}^{\mathrm{P}}$ denotes a weighted extrinsic curvature associated with the linear momentum of a $\mathrm{BH}$;

$$
K_{i j}^{\mathrm{P}}=\frac{3}{2 r_{\mathrm{BH}}^{2}}\left(n_{i} P_{j}+n_{j} P_{i}+\left(n_{i} n_{j}-\delta_{i j}\right) P_{k} n^{k}\right) .
$$

Here, $n_{k}=n^{k}=x_{\mathrm{BH}}^{k} / r_{\mathrm{BH}}$ and $P_{i}\left(=P^{i}\right)$ denotes the linear momentum of the $\mathrm{BH}$, determined from the condition that the total linear momentum of system is zero as

$$
P_{i}=-\int J_{i} \psi^{6} d^{3} x
$$

The right-hand side of Eq. (10) denotes the linear momentum of the companion NS. Then, the total angular momentum of the system is derived from

$$
J=\int J_{\varphi} \psi^{6} d^{3} x+\epsilon_{z j k} x_{\mathrm{P}}^{j} P^{k}
$$

where we assume that the $z$ axis is the axis of orbital rotation.

Substituting Eq. (8) into Eq. (3), an elliptic equation for $W_{i}$ is derived in the form

$$
\Delta W_{i}+\frac{1}{3} \partial_{i} \partial_{k} W^{k}=8 \pi J_{i} \psi^{6} .
$$

Denoting $W_{i}=7 B_{i}-\left(\chi_{, i}+B_{k, i} x^{k}\right)$ where $\chi$ and $B_{i}$ are auxiliary functions, we decompose Eq. (12) into two linear elliptic equations

$$
\Delta B_{i}=\pi J_{i} \psi^{6} \text { and } \Delta \chi=-\pi J_{i} x^{i} \psi^{6} .
$$

To determine a BH-NS binary in quasiequilibrium states, in addition, we have to determine the shift vector, $\beta^{i}$. The reason for this is that $\beta^{i}$ appears in the hydrostatic equations [23]. In the conformal-flatness formalism, the relation between $\beta^{i}$ and $\hat{A}_{i j}$ is written as

$$
\delta_{j k} \partial_{i} \beta^{k}+\delta_{i k} \partial_{j} \beta^{k}-\frac{2}{3} \delta_{i j} \partial_{k} \beta^{k}=\frac{2 \alpha}{\psi^{6}} \hat{A}_{i j} .
$$

Operating $\delta^{j l} \partial_{l}$ to this equation, an elliptic equation is derived

$$
\Delta \beta^{i}+\frac{1}{3} \delta^{i k} \partial_{k} \partial_{j} \beta^{j}=2 \partial_{j}\left(\alpha \psi^{-6}\right) \hat{A}^{i j}+16 \pi \alpha J_{j} \delta^{i j} .
$$

For $\hat{A}_{i j}$ in the right-hand side of this equation, we substitute the relation of Eq. (8) [not Eq. (14)]. As a result, no singular term appears in the right-hand side of Eq. (15), and thus, $\beta^{i}$ is solved in the same manner as that for $W_{i}$.

In this formulation, the elliptic equations for the gravitational-field components, $\phi, \eta, B_{i}, \chi$, and $\beta^{i}$, and the velocity potential have to be solved. For a numerical solution of them, we use the LORENE library [28], by which a high-precision numerical solution can be computed using the spectral method. We note that in the 
puncture framework, we do not basically have to impose an inner boundary condition around the BH. In this point, the moving-puncture framework differs from the excision framework, and this may be a demerit of the moving-puncture framework because a physical condition may not be imposed for the BH. However, this may be also a merit of this framework because there remains a degree of freedom which can be used to adjust the property of the quasiequilibrium to a desired state, as mentioned in the following.

The final remaining task in the moving-puncture framework is to determine the center of mass of system. The issue in this framework is that we do not have any natural physical condition for determining it. (By contrast, the condition is automatically derived in the excision framework [7], although it is not clear whether the condition is really physical and whether the resulting quasiequilibrium is a quasicircular state; see, e.g., Ref. [30] which assesses the circularity of the quasiequilibrium.) In our first paper [13], we employed a condition that the dipole part of $\psi$ at spatial infinity is zero. However, in this method, the angular momentum derived for a close orbit of $m_{0} \Omega \gtrsim 0.03$ is by $\sim 2 \%$ smaller than that derived by the third post-Newtonian (3PN) approximation [29]. (Note $m_{0}=M_{\mathrm{BH}}+M_{\mathrm{NS}}$.) Because the $3 \mathrm{PN}$ approximation should be an excellent approximation of general relativity for describing a binary of a fairly distant orbit as $m_{0} \Omega \approx 0.03$, we should consider that the obtained initial data deviates from the true quasicircular state, and thus, the initial orbit would be eccentric. Such initial condition is not suitable for quantitatively accurate numerical simulation of the inspiraling BH-NS binaries.

In the subsequent work [14], we adopted a condition that the azimuthal component of the shift vector $\beta^{\varphi}$ at the location of the puncture $\left(\boldsymbol{r}=\boldsymbol{r}_{\mathrm{P}}\right)$ is equal to $-\Omega$; i.e., we imposed a corotating gauge condition at the location of the puncture. In the following, we refer to this condition as " $\beta^{\varphi}$ condition". This is slightly better than the original condition, but the angular momentum derived for a close orbit of $m_{0} \Omega \gtrsim 0.03$ is still by $\gtrsim 1 \%$ smaller than that derived by the $3 \mathrm{PN}$ relation for a large mass ratio $Q \geq 2$ (see Ref. [23] for detailed numerical results). The disagreement is larger for the larger mass ratio. As a result of this, the initial condition is likely to deviate from the true quasicircular state and hence the initial orbital eccentricity is not also negligible (see Sec. IV C of Ref. [16] for numerical evolution of such initial data), in particular, for binaries of large mass ratios. This also suggests that the $\beta^{\varphi}$ condition is not suitable for deriving a realistic quasicircular state.

If the simulations are started with a sufficiently large orbital separation, the eccentricity, which is initially $\sim 0.1$, will decrease to $\sim 0.01$ within several orbits, because gravitational radiation reaction has a strong effect to reduce the orbital eccentricity 31]. However, the separation has to be large enough for the large initial eccentricity to be reduced. This implies that a long-term simulation, which is not computationally favored, is required. (As we illustrate in this paper, the eccentricity is not sufficiently suppressed to $\lesssim 0.01$ in $\sim 5$ orbits if we adopt the initial condition obtained in the $\beta^{\varphi}$ condition.)

In this paper, we employ a new condition in which the center of mass is determined in a phenomenological manner: We impose the condition that the total angular momentum of the system for a given value of $m_{0} \Omega$ agrees with that derived by the $3 \mathrm{PN}$ approximation (see Ref. 32] for a similar concept). This can be achieved by appropriately choosing a hypothetical position of the center of mass (it may not be better to refer to this position as "the center of mass" but simply as "the rotation axis"). With this method, the total energy of the system does not agree completely with that derived by the $3 \mathrm{PN}$ approximation [23], and thus, the eccentricity does not become zero. However, the resulting eccentricity in this condition is much smaller than that in the $\beta^{\varphi}$ condition (see Fig. 1), and thus, for a moderately long-term simulation (in $\sim 2-3$ orbits), the effect of the eccentricity is suppressed to an acceptable level for a scientific discussion, as shown in Sec. IV] We refer to this condition as "3PN-J condition" in the following.

Figure 1 plots evolution of the orbital separation for $\mathcal{C}=0.145$ and for $Q=2$ and 5 . Here, the separation is defined as the coordinate separation between the positions of the puncture and of the maximum density of the NS, $r_{\text {sep }}=\left|x_{\text {sep }}^{i}\right|$; see Eq. (21). For the results with the initial condition derived in the $\beta^{\varphi}$ condition, the separation badly oscillates with time and the amplitude of this oscillation is still conspicuous even after 3-4 orbital motions. Consequently, the eccentricity is not negligible even at the last orbit just before the merger. By contrast, for the case that the initial condition is derived in the $3 \mathrm{PN}-\mathrm{J}$ condition, the amplitude of the oscillation is much smaller and, furthermore, the amplitude of this oscillation does not become conspicuous within about two orbital motions. Although the eccentricity does not become exactly zero, it is within an acceptable level at the last orbit just before the merger (see, e.g. gravitational waveforms shown in Sec. IV C).

We note that the coordinate separation is a gaugedependent quantity and hence the discussion here is not based on the very physical quantity. The physical quantity such as the orbital eccentricity is not rigously extracted from it. However, the similar quantitative feature is seen if we plot evolution of the gravitational-wave frequency as a function of time (which is the physical quantity); the oscillation amplitude of the orbital separation shows the magnitude of the orbital eccentricity at least approximately (see Sec. IVC for more physical analysis).

\section{B. Chosen models}

In the polytropic EOS, the adiabatic constant, $\kappa$, is a free parameter, and thus, physical units such as mass, 


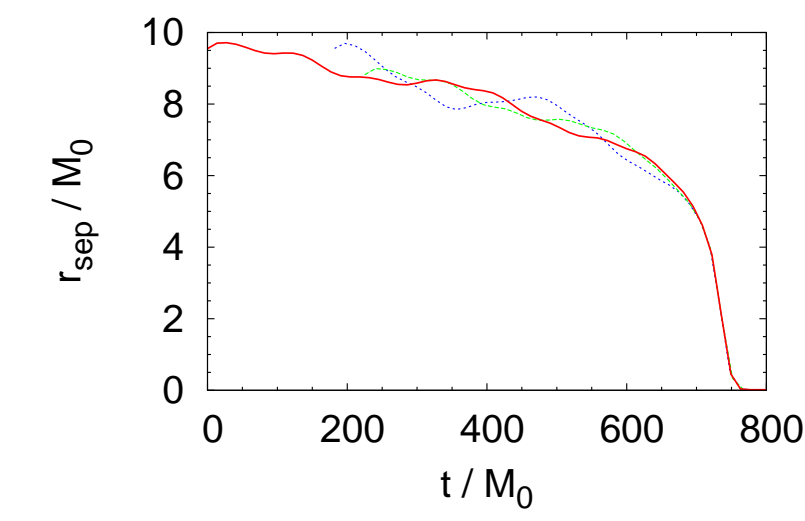

(a)

1: Evolution of orbital separation (a) for model M20.145 and (b) for model M50.145. The solid and dotted curves denote the results with the initial conditions obtained in the 3PN-J and $\beta^{\varphi}$ conditions, respectively. The dashed curve in panel (a) denote the result for model M20.145N. To align the curves at the onset of the merger, the time is appropriately shifted for the results of M20.145N, M20.145b, and M50.145b. Note that the merger sets in when the orbital separation becomes $\sim 5 M_{0}$. The binaries for models M20.145 and M50.145 spend in the inspiral phase for $\sim 5$ and 7.5 orbits, respectively (see Sec. IV), whereas those for models M20.145b and M50.145b spend only for $\sim 4$ and 5.25 orbits, respectively.

TABLE I: Key parameters for the initial conditions adopted for the numerical simulation in units of $\kappa=1$. The mass ratio $\left(Q=M_{\mathrm{BH}} / M_{\mathrm{NS}}\right), \mathrm{BH}$ mass $\left(M_{\mathrm{BH}}\right)$, mass parameter of the puncture $\left(M_{\mathrm{p}}\right)$, rest mass of the NS $\left(M_{*}\right)$, mass $\left(M_{\mathrm{NS}}\right)$ and compactness $\left(\mathcal{C}=M_{\mathrm{NS}} / R_{\mathrm{NS}}: R_{\mathrm{NS}}\right.$ is the circumferential radius) of the NS when it is in isolation, maximum density of the NS $\left(\rho_{\max }\right), \mathrm{ADM}$ mass of the system $\left(M_{0}\right)$, total angular momentum $\left(J_{0}\right)$ in units of $M_{0}^{2}$, orbital period $\left(P_{0}\right)$ in units of $M_{0}$, and orbital angular velocity $\left(\Omega_{0}\right)$ in units of $m_{0}^{-1}$. The first and second numerical values described for the model name (the first column) denote the values of $Q$ and $\mathcal{C}$, respectively. The first 10 models are computed in the 3PN-J condition and the last 4 are in the $\beta^{\varphi}$ condition.

\begin{tabular}{cccccccccccc}
\hline $\begin{array}{c}\text { 3PN-J } \\
\text { Model }\end{array}$ & $Q$ & $M_{\mathrm{BH}}$ & $M_{\mathrm{p}}$ & $M_{*}$ & $M_{\mathrm{NS}}$ & $M_{\mathrm{NS}} / R_{\mathrm{NS}}$ & $\rho_{\max }$ & $M_{0}$ & $J_{0} / M_{0}^{2}$ & $P_{0} / M_{0}$ & $m_{0} \Omega_{0}$ \\
\hline M15.145 & 1.5 & 0.2093 & 0.2043 & 0.1500 & 0.1395 & 0.145 & 0.1262 & 0.3452 & 0.8393 & 212.5 & 0.02987 \\
\hline M20.145 & 2 & 0.2790 & 0.2736 & 0.1500 & 0.1395 & 0.145 & 0.1261 & 0.4146 & 0.8603 & 213.7 & 0.02968 \\
\hline M20.145N & 2 & 0.2790 & 0.2732 & 0.1500 & 0.1395 & 0.145 & 0.1260 & 0.4143 & 0.8418 & 191.8 & 0.03309 \\
\hline M20.160 & 2 & 0.2957 & 0.2900 & 0.1600 & 0.1478 & 0.160 & 0.1512 & 0.4394 & 0.8608 & 214.4 & 0.02958 \\
\hline M20.178 & 2 & 0.3119 & 0.3059 & 0.1700 & 0.1560 & 0.178 & 0.1890 & 0.4635 & 0.8582 & 211.4 & 0.03001 \\
\hline M30.145 & 3 & 0.4185 & 0.4121 & 0.1500 & 0.1395 & 0.145 & 0.1255 & 0.5534 & 0.7091 & 192.2 & 0.03298 \\
\hline M30.160 & 3 & 0.4435 & 0.4367 & 0.1600 & 0.1478 & 0.160 & 0.1504 & 0.5864 & 0.7096 & 192.9 & 0.03285 \\
\hline M30.178 & 3 & 0.4679 & 0.4608 & 0.1700 & 0.1560 & 0.178 & 0.1878 & 0.6187 & 0.7103 & 193.8 & 0.03269 \\
\hline M40.145 & 4 & 0.5580 & 0.5512 & 0.1500 & 0.1395 & 0.145 & 0.1252 & 0.6926 & 0.6042 & 192.1 & 0.03294 \\
\hline M50.145 & 5 & 0.6975 & 0.6905 & 0.1500 & 0.1395 & 0.145 & 0.1249 & 0.8318 & 0.5238 & 191.8 & 0.03296 \\
\hline$\beta^{\varphi}$ & & & & & & & & & & & \\
Model & $Q$ & $M_{\mathrm{BH}}$ & $M_{\mathrm{p}}$ & $M_{*}$ & $M_{\mathrm{NS}}$ & $M_{\mathrm{NS}} / R_{\mathrm{NS}}$ & $\rho_{\max }$ & $M_{0}$ & $J_{0} / M_{0}^{2}$ & $P_{0} / M_{0}$ & $m_{0} \Omega_{0}$ \\
\hline M20.145b & 2 & 0.2790 & 0.2737 & 0.1500 & 0.1395 & 0.145 & 0.1261 & 0.4144 & 0.8462 & 211.0 & 0.03007 \\
\hline M30.145b & 3 & 0.4185 & 0.4121 & 0.1500 & 0.1395 & 0.145 & 0.1256 & 0.5531 & 0.6960 & 189.5 & 0.03345 \\
\hline M40.145b & 4 & 0.5580 & 0.5512 & 0.1500 & 0.1395 & 0.145 & 0.1252 & 0.6922 & 0.5905 & 188.9 & 0.03351 \\
\hline M50.145b & 5 & 0.6975 & 0.6905 & 0.1500 & 0.1395 & 0.145 & 0.1250 & 0.8315 & 0.5134 & 189.1 & 0.03345 \\
\hline & & & & & & & & & & &
\end{tabular}

radius, and time can be rescaled arbitrarily by simply changing the value if $\kappa$ : i.e., when a numerical result for a particular value (say $\kappa=\kappa_{1}$ ) is obtained, we can also obtain the numerical results of these quantities for $\kappa=\kappa_{2}$ by simply rescaling them by a factor of $\left(\kappa_{2} / \kappa_{1}\right)^{1 / 2(\Gamma-1)}$. This implies that $\kappa$ can be completely scaled out of the problem. In this paper, we present the results in units of $\kappa=1$ (and $c=G=1$ ), because such units are popular in other groups [15, 17]. In the polytropic EOS, the nondimensional quantities such as $\mathcal{C}=G M_{\mathrm{NS}} / c^{2} R_{\mathrm{NS}}$, $M_{0} \Omega, M_{0} \kappa^{-1 / 2(\Gamma-1)}, R_{\mathrm{NS}} \kappa^{-1 / 2(\Gamma-1)}$, and mass ratio $(Q)$ are unchanged irrespective of the value of $\kappa$ and have an invariant meaning.

In the present work, the numerical simulation is per- 


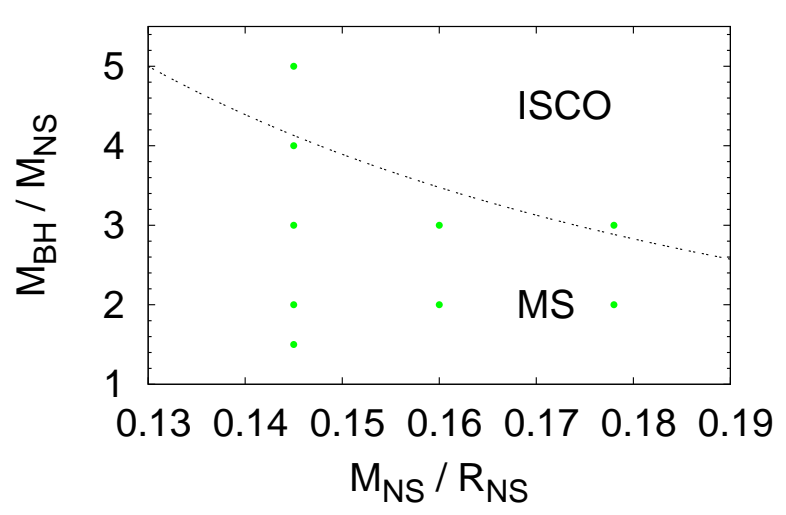

FIG. 2: Initial conditions in the parameter space of $(\mathcal{C}, Q)$ are plotted by the solid circles. The meaning of the dashed curve is as follows [7]: For the binaries located above the dashed curve, mass-shedding (MS) does not occur until the binaries reach the ISCO, whereas for the binaries below the dashed curve, the mass-shedding will occur for the NS due to the tidal force of the BH before the ISCO is reached.

formed for a wide variety of initial conditions (see Table I), but for restricting that the $\mathrm{BH}$ spin is zero. We characterize the BH-NS binaries by the mass ratio, $Q=M_{\mathrm{BH}} / M_{\mathrm{NS}}$, and the compactness of the NS, $\mathcal{C}=M_{\mathrm{NS}} / R_{\mathrm{NS}}$. (Note that in Refs. [14, 16], we use $q=1 / Q$ instead of $Q$ to specify the model.) The mass ratio is chosen in the range between 1.5 and 5.0, and the compactness of the NS is in the range, $0.145-0.178$. We note that the typical mass of the NS in nature is 1.3$1.4 M_{\mathrm{NS}}[33]$ and the likely lower bound of the $\mathrm{BH}$ mass is $\sim 2 M_{\odot}$. This implies that $Q$ should be chosen to be larger than $\sim 1.5$. The circumferential radius of an NS of $M_{\mathrm{NS}}=1.35 M_{\odot}$ and $\mathcal{C}=0.145,0.160$, and 0.178 is $13.8,12.5$, and $11.2 \mathrm{~km}$, which are reasonable values for modeling the NSs.

Figure 2 shows the initial conditions in the parameter space of $(\mathcal{C}, Q)$. The meaning of the dashed curve, derived in Ref. [7], is as follows: For the binaries located above the dashed curve, mass-shedding of the NS by the tidal effect of the companion $\mathrm{BH}$ does not occur until the binary reaches the ISCO, whereas for the binary shown below the dashed curve, the mass-shedding will occur for the NS before the ISCO is reached and in such a system, tidal disruption may subsequently occur. Thus, many of the NSs in the chosen binary system will be subject to the tidal effect of the companion $\mathrm{BH}$ in a close orbit, but for some of them (e.g., $(\mathcal{C}, Q)=(0.145,5)$ and $(0.178$, $3)$ ), the tidal effect is unlikely to play an essential role in the inspiral phase. We also note that an NS which is in the mass-shedding is not always tidally disrupted immediately, although the mass-shedding NS is the candidate to be tidally disrupted. Namely, the condition for inducing the tidal disruption is in general more restricted than that for inducing the mass-shedding (see Sec. IV).

In the present work, we prepare quasiequilibrium states with $m_{0} \Omega_{0} \approx 0.030$ for $Q=1.5$ and 2 , and $m_{0} \Omega_{0} \approx 0.033$ for $Q=2-5$, where $\Omega_{0}$ is the orbital angular velocity. We choose these values of $\Omega_{0}$ so as for the BH-NS binaries to experience more than 4 orbits before the onset of the merger. In such a long-term evolution, the eccentricity which presents at $t=0$ decreases to an acceptable level during the inspiral phase, and also the nonzero radial velocity associated with the gravitational radiation reaction is approximately taken into account from the late inspiral phase.

In Table I, several key quantities for the quasiequilibrium states adopted in this paper are listed. Specifically, we prepare 10 models. The first two and last three numerical numbers described for the model name denote the values of $Q$ and $\mathcal{C}$, respectively. For comparison, the same quantities are also listed for 4 selected quasiequilibrium states obtained in the $\beta^{\varphi}$ condition. We find that the angular momentum of them is by $\sim 2 \%$ smaller than that of the quasiequilibrium states obtained in the 3PN-J condition for $m_{0} \Omega_{0} \approx 0.033$.

\section{PREPARATION FOR NUMERICAL SIMULATION}

\section{A. Brief summary of formulation and methods}

The numerical simulations are performed using a code SACRA recently developed in our group [16]. The details of the chosen scheme, formulation, gauge condition, and methods for the analysis are described in Ref. [16] to which the reader may refer. Reference [16] also shows that SACRA can successfully simulate the inspiral and merger of BH-BH, NS-NS, and BH-NS binaries.

In SACRA, the Einstein equations are solved in a moving-puncture version 18, 19, 26] of the BaumgarteShapiro-Shibata-Nakamura formalism [34]. Specifically, we evolve $W \equiv \gamma^{-1 / 6}$ [35], conformal three-metric, $\tilde{\gamma}_{i j} \equiv \gamma^{-1 / 3} \gamma_{i j}$, the tracefree extrinsic curvature, $\tilde{A}_{i j} \equiv$ $\gamma^{-1 / 3}\left(K_{i j}-K \gamma_{i j} / 3\right)$, the trace part of $K_{i j}, K$, and a three auxiliary variable, $\Gamma^{i} \equiv-\partial_{j} \tilde{\gamma}^{i j}$ (or $F_{i} \equiv \partial_{j} \tilde{\gamma}_{i j}$ ). Here, $\gamma_{i j}$ is the three-metric, $K_{i j}$ the extrinsic curvature, and $\gamma \equiv \operatorname{det}\left(\gamma_{i j}\right)$. In the numerical simulation, a fourthorder finite differencing scheme in space and time is used implementing an adaptive mesh refinement (AMR) algorithm (at refinement boundaries, a second-order interpolation scheme is partly adopted). The advection term such as $\beta^{i} \partial_{i} \tilde{\gamma}_{j k}$ is evaluated by a fourth-order noncentered finite-difference, as proposed in Ref. [26].

The moving-puncture approach is adopted for evolving the BH [19] (see also Ref. [36]); i.e., we adopt one of the moving-puncture gauge conditions as [26]

$$
\begin{aligned}
& \left(\partial_{t}-\beta^{j} \partial_{j}\right) \beta^{i}=0.75 B^{i}, \\
& \left(\partial_{t}-\beta^{j} \partial_{j}\right) B^{i}=\left(\partial_{t}-\beta^{j} \partial_{j}\right) \tilde{\Gamma}^{i}-\eta_{s} B^{i},
\end{aligned}
$$

where $B^{i}$ is an auxiliary variable and $\eta_{s}$ is an arbitrary constant. In the present paper, we set $\eta_{s}=0.414$ in units of $\kappa=c=G=1$. 
TABLE II: Parameters of the grid structure for the numerical simulation with our AMR algorithm. In the column named "Levels", the number of total refinement levels is shown (in the bracket, the numbers of coarser and finer levels are specified; see Ref. [16] for the definition of the coarser and finer levels). $\Delta x\left(=h_{7}\right)$ is the minimum grid spacing for $N=36, R_{\text {diam }}$ the coordinate length of semi-major diameter of the NS, $L$ the location of outer boundaries along each axis, $\lambda_{0}\left(=\pi / \Omega_{0}\right)$ the gravitational wavelength at $t=0$, and $\Delta x_{\mathrm{gw}}$ the grid spacing, by which gravitational waves are extracted for $N=36$. Note that $M_{\mathrm{BH}}$ denotes the $\mathrm{BH}$ mass (the irreducible mass) at $t=0$. The grid structures for models M20.145b-M50.145b are the same as those for models M20.145-M50.145, respectively. For the simulations with $N \neq 36$, the size of each domain (and hence $L)$ is unchanged, and thus, the grid spacing $h_{l}$ is simply changed.

\begin{tabular}{cccccc}
\hline Run & Levels & $\Delta x / M_{0}\left(\Delta x / M_{\mathrm{BH}}\right)$ & $R_{\text {diam }} / \Delta x$ & $L / M_{0}\left(L / \lambda_{0}\right)$ & $\Delta x_{\mathrm{gw}} / M_{0}$ \\
\hline M15.145 & $8(4+4)$ & $0.0407(0.0672)$ & 112 & $187.7(1.77)$ & $1.30-5.21$ \\
\hline M20.145 & $8(4+4)$ & $0.0377(0.0560)$ & 99.3 & $173.7(1.63)$ & $1.21-4.82$ \\
\hline M20.145N & $8(4+4)$ & $0.0377(0.0560)$ & 99.4 & $173.8(1.81)$ & $1.21-4.83$ \\
\hline M20.160 & $8(4+4)$ & $0.0356(0.0528)$ & 92.9 & $163.9(1.53)$ & $1.14-4.55$ \\
\hline M20.178 & $8(4+4)$ & $0.0324(0.0481)$ & 89.1 & $149.1(1.41)$ & $1.04-4.14$ \\
\hline M30.145 & $8(4+4)$ & $0.0305(0.0403)$ & 90.0 & $140.5(1.46)$ & $0.98-3.90$ \\
\hline M30.160 & $8(3+5)$ & $0.0266(0.0352)$ & 91.2 & $122.8(1.27)$ & $0.85-3.41$ \\
\hline M30.178 & $8(3+5)$ & $0.0253(0.0334)$ & 84.1 & $116.3(1.20)$ & $0.81-3.23$ \\
\hline M40.145 & $8(3+5)$ & $0.0244(0.0302)$ & 89.0 & $112.3(1.17)$ & $0.78-3.12$ \\
\hline M50.145 & $8(3+5)$ & $0.0203(0.0242)$ & 88.4 & $93.5(0.98)$ & $0.65-2.60$ \\
\hline
\end{tabular}

For the numerical hydrodynamics, we evolve $\rho_{*} \equiv$ $\rho \alpha u^{t} e^{6 \phi}, \hat{u}_{i} \equiv h u_{i}$, and $e_{*} \equiv \rho h \alpha u^{t}-P /\left(\rho \alpha u^{t}\right)$. To handle the advection terms in the hydrodynamic equations, a high-resolution central scheme [37] is adopted with a third-order piecewise parabolic interpolation and with a steep min-mod limiter in which the limiter parameter $b$ is set to be 3 (see appendix A of Ref. 38]). We adopt the $\Gamma$-law EOS in the simulation as

$$
P=(\Gamma-1) \rho \varepsilon
$$

where $\Gamma=2$.

Properties of the BHs such as mass and spin are determined by analyzing area and circumferential radii of apparent horizons. A numerical scheme of our apparent horizon finder is described in Refs. [16, 39].

Gravitational waves are computed by extracting the outgoing part of the Newman-Penrose quantity (the socalled $\Psi_{4}$ ). The extraction of $\Psi_{4}$ is carried out for several constant coordinate radii, $r \approx 50-100 M_{0}$. The plus and cross modes of gravitational waves are obtained by performing time integration of $\Psi_{4}$ twice, with appropriate choice of integration constants and subtraction of unphysical drift which is caused primarily by the drift of the center of mass of the system. (Because we extract $\Psi_{4}$ for fixed, finite coordinate radii, the drift of the center of mass spuriously affects gravitational waveforms.) Specifically, whenever the time integration is performed, we subtract a function of the form $a_{2} t^{2}+a_{1} t+a_{0}$ where $a_{0}-$ $a_{2}$ denote constants which are determined by the leastsquare fitting of the numerical data.

We compute the modes of $2 \leq l \leq 4$ and $|m| \leq l$ for $\Psi_{4}$, and found that the quadrupole mode of $(l,|m|)=(2,2)$ is always dominant, but $(l,|m|)=(3,3),(4,4)$, and $(2$, 1) modes also contribute to the energy and angular momentum dissipation by more than $1 \%$ for some of models (in particular for large values of $Q$; cf. Table IV).

We also estimate the kick velocity from the linear momentum flux of gravitational waves. The linear momentum flux $d P_{i} / d t$ is computed by the same method as that given in, e.g., Refs. [26, 40]. Specifically, the coupling terms between $(l, m)=(2, \pm 2)$ and $(3, \mp 3)$ modes, between $(2, \pm 2)$ and $(2, \mp 1)$ modes, and between $(3, \pm 3)$ and $(4, \mp 4)$ modes contribute primarily to the linear momentum flux [41]. From the total linear momentum dissipated by gravitational waves,

$$
\Delta P_{i}=\int \frac{d P_{i}}{d t} d t
$$

the kick velocity is defined by $\Delta P_{i} / M_{0}$ where $M_{0}$ is the initial ADM mass of the system.

\section{B. Setting grids for AMR scheme}

The Einstein and hydrodynamic equations are solved in an AMR algorithm described in Ref. [16]. In the present work, we prepare 8 refinement-level domains of different grid resolutions and domain sizes. Each domain is composed of the uniform vertex-center-grid with grid number $(2 N+1,2 N+1, N+1)$ for $(x, y, z)$ where $N$ is a constant and is always chosen to be 24,30 , and 36 to check convergence of numerical results. The equatorial plane symmetry with respect to the $z=0$ plane is assumed. The length of a side for the largest domain is denoted by $2 L$, and the grid spacing for each domain is $h_{l}=L / N / 2^{l}$ for $l=0-7$. As described in Ref. [16], the regions around a $\mathrm{BH}$ and an NS are covered by "finer" domains which move with the $\mathrm{BH}$ and NS. On the other hand, "coarser" domains which cover wider regions do not move and their center is fixed approximately to be the center of mass of the system. 
Table II lists the parameters for the grid structure in our AMR scheme. $\lambda_{0}$ denotes the wavelength of gravitational waves at $t=0\left(\lambda_{0}=\pi / \Omega_{0}\right)$. For all the cases, $L$ is chosen to be $1-2 \lambda_{0}$, implying that the outer boundaries are located in a wave zone. The NS is covered by the finest and second-finest domains and the coordinate radius of the apparent horizon for the $\mathrm{BH}$ is always covered by more than 15 grid points for $N=36$. Because the numerical results (such as the time spent in the inspiral phase, the mass and spin for the final state of the $\mathrm{BH}$, and total energy radiated by gravitational waves) for $N \geq 30$ depend only weakly on the grid resolution, we conclude that the convergence is approximately achieved for $N=36$ (except for the rest mass of disks which are formed for the model with small values of $\mathcal{C}$ and $Q$; see Sec. IVA).

For $N=36$, the total memory required for the simulation with 13 domains is about 5 GBytes. We perform all the simulations using personal computers of 8 GBytes memory and 2-8 processors (in one job, only two processors are used with an OpenMP library). The typical computation time for one model with $N=36$ is $5-7$ weeks on the personal computers of clock speed $3 \mathrm{GHz}$.

\section{Atmosphere}

Because any conservation scheme of hydrodynamics is unable to evolve a vacuum, we have to introduce an artificial atmosphere outside the NSs. The density of the atmosphere has to be small enough to exclude its spurious effect to the orbital motion of the $\mathrm{BH}$ and NS, and to avoid overestimation of the total rest mass for a disk surrounding the $\mathrm{BH}$, which may be formed after the merger if the NS is tidal disrupted. We initially assign a small rest-mass density for the atmosphere as follows

$$
\rho_{\text {atmo }}= \begin{cases}\rho_{\text {crit }} & r \leq r_{0} \\ \rho_{\text {crit }} e^{1-r / r_{0}} & r>r_{0} .\end{cases}
$$

where $r_{0}$ is a constant chosen to be $5 L / 32$. We choose $\rho_{\text {crit }}=\rho_{\max } \times 10^{-9}$ where $\rho_{\max }$ is the maximum restmass density of the NS initially given. With such choice, the total amount of the rest mass in the atmosphere is less than $10^{-5} M_{*}$. Accretion of the atmosphere of such small mass onto the $\mathrm{BH}$ and NS plays a negligible role for their orbital evolution. As we state in Sec. I, one of the important tasks for the merger simulation of the BH-NS binaries is to determine the rest mass of disks surrounding a $\mathrm{BH}$ formed after the merger. In the simulation, we pay attention only to the case that the disk mass is larger than $10^{-5} M_{*}$.

During the evolution, we also adopt an artificial treatment for the low-density region in the following manner: (i) If the density is smaller than $\rho_{\text {atmo }}$, we set $\rho=\rho_{\text {atmo }}$ and $u_{i}=0$. Then, the specific internal energy $\varepsilon$ is set to be $\kappa \rho_{\text {crit }}^{\Gamma-1} /(\Gamma-1)$. (ii) Even if the density is larger than $\rho_{\text {atmo }}$, we reduce the specific momentum $h u_{i}$ by a factor of $1-\exp \left[-\rho / \rho_{\text {crit }}\right]$; i.e., for a fluid of density $\lesssim 5 \rho_{\text {crit }}$, the specific momentum is artificially reduced. The reason that we adopt these treatments is that a numerical instability resulting in a negative density or pressure often happens accidentally for the low-density region in the absence of the artificial treatment. However, by limiting the unphysical growth of the specific momentum, as described above, such instabilities are excluded. For some models, we checked whether the magnitude of $\rho_{\text {crit }}$ affects the numerical result, but as long as the small value is chosen for it as in the present work, dependence of the numerical results on the magnitude of $\rho_{\text {crit }}$ is quite tiny.

\section{NUMERICAL RESULTS}

\section{A. Orbital evolution, tidal disruption, and disk mass}

Figure 3 plots evolution of the coordinate separation between the $\mathrm{BH}$ and $\mathrm{NS}, x_{\mathrm{sep}}^{i}$, for models M20.145, M40.145, and M50.145. $x_{\text {sep }}^{i}$ is defined by

$$
x_{\mathrm{sep}}^{i}=x_{\mathrm{NS}}^{i}-x_{\mathrm{BH}}^{i},
$$

where $x_{\mathrm{NS}}^{i}$ and $x_{\mathrm{BH}}^{i}$ denote the positions of the maximum rest-mass density of the NS and of the puncture, respectively. This figure illustrates that the binaries are in a slightly eccentric orbit for the first $\sim 2$ orbits because the strictly circular orbit is not provided initially. Also, in the first $\sim 2$ orbits, decrease rate of the orbital separation due to gravitational radiation reaction is not as large as that predicted by the $\mathrm{PN}$ theory, in particular for larger values of $Q$. However, because the eccentricity decreases due to the gravitational radiation reaction during the evolution and also the initial eccentricity is not very large (see, e.g., Fig. 1), the orbit approaches approximately to a quasicircular orbit after a few orbits. The resulting final $2-3$ orbits before the onset of merger appear to be close to a quasicircular one. This behavior is much better than that obtained when an initial condition computed in the $\beta^{\varphi}$ condition is adopted (see, e.g. Fig. 15 of Ref. [16]).

For models M20.145, M40.145, and M50.145, the binaries spend in the inspiral phase for $\sim 4.8,6.5$, and 7.8 orbits, respectively. For the larger values of $Q$ with an approximately fixed value of $m_{0} \Omega_{0}$, the number of the inspiral orbit is larger, because the luminosity of gravitational waves is approximately proportional to $Q^{2} /(1+Q)^{4}$, and as a result, the binary evolves more slowly for the larger values of $Q$.

Figures 4, 6] plot late-time evolution of the rest-mass density contour curves and density contrasts as well as the location of the BHs for models M20.145, M40.145, and M50.145, respectively. For model M20.145, the NS is tidally disrupted in the late inspiral phase (see the first panel of Fig. 4). Subsequently the material of the NS forms a one-armed spiral arm around its companion $\mathrm{BH}$ (second panel of Fig. 4). In the spiral arm, a 


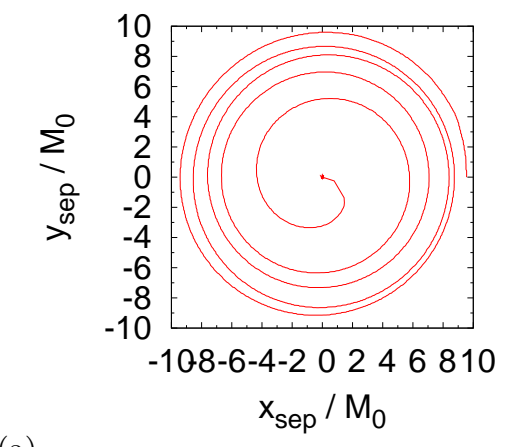

(a)

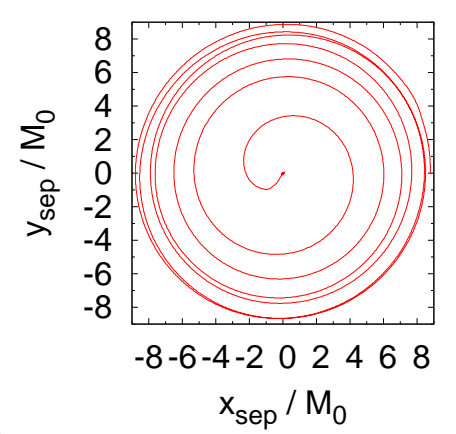

(b)

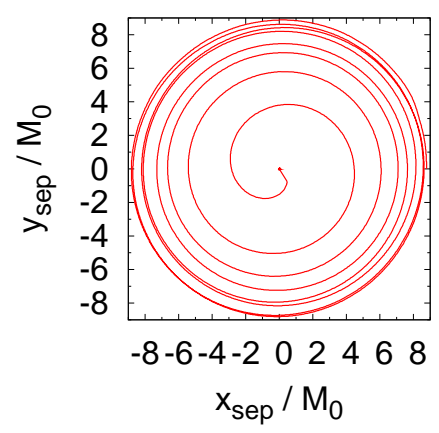

FIG. 3: Coordinate separation between the BH and NS (a) for models M20.145, (b) M40.145, and (c) M50.145. Here, $r_{\mathrm{sep}}=\left|x_{\mathrm{NS}}^{i}-x_{\mathrm{BH}}^{i}\right|$; see Eq. (21).

FIG. 4: Snapshots of the density contour curves and density contrasts as well as the location of the BH in the merger and ringdown phases for model M20.145. The contour curves are plotted for $\rho=10^{-i}$ where $i=2,3,4,5$ in the first four panels, whereas in the last two, $\rho=10^{-i}$ where $i=3,4$, and 5 . The first panel denotes the state just at the onset of the merger. The filled circles show the region inside the apparent horizon. Note that the maximum value of $\rho$ for the NS in the inspiral phase is $\approx 0.126$.

transport process of angular momentum from its inner to outer region is likely to work efficiently. Then, the spiral arm winds around the BH (third panel of Fig. 4), and most of the fluid elements, which do not have angular momentum large enough to have an orbit around the $\mathrm{BH}$, fall into the $\mathrm{BH}$. In the first $\sim 200 M_{0}$ after the tidal disruption, $\sim 98 \%$ of the material falls into the $\mathrm{BH}$ (see Fig. 7). However, a small fraction of the fluid elements obtain angular momentum large enough to escape the capture by the $\mathrm{BH}$, and form a disk around the $\mathrm{BH}$. For $t-t_{\text {merger }} \gtrsim 300 M_{0}$, where $t_{\text {merger }}$ approximately denotes the time at which the merger sets in, accretion rate decreases, and then, the disk relaxes to a quasisteady state (fourth-sixth panels). For model M20.145, the rest mass of the disk is $\sim 0.01 M_{*}$ at $t-t_{\text {merger }} \approx 1000 M_{0}$ for $N=36$ (cf. Fig. 7). For a hypothetical value of $M_{\mathrm{NS}}=1.35 M_{\odot}, 1000 M_{0}$ is approximately equal to 20 $\mathrm{ms}$, and thus, the lifetime of the formed disk is likely to be much longer than $20 \mathrm{~ms}$. Also, for a hypothetical value of $M_{\mathrm{NS}}=1.35 M_{\odot}, \rho=10^{-4}$ in the units of $c=G=\kappa=1$ corresponds to $\rho \approx 6.0 \times 10^{11} \mathrm{~g} / \mathrm{cm}^{3}$. Thus, for that hypothetical mass, the rest-mass density of the disk is high as $\sim 10^{11}-10^{12} \mathrm{~g} / \mathrm{cm}^{3}$. Evolution and the final outcome for model M15.145 are similar to those for model M20.145, although the disk mass is by a factor of $\sim 2$ larger due to the smaller value of $Q$.

The NS for model M40.145 is also subject to tidal deformation and mass shedding by the tidal effects of the companion $\mathrm{BH}$ in the late inspiral phase, as indicated in Fig. 2 and in the first panel of Fig. 5. However, the tidal effects in this binary become important only for the inspiral orbits close to the ISCO. Because the approaching velocity is a substantial fraction of the orbital velocity at such close orbits, the time scale available for the NS to be tidally deformed before the onset of the merger is too short to efficiently transport angular momentum inside the NS. As a result, one-armed spiral arm is formed in a less conspicuous manner than that for model M20.145 (see the second panel of Fig. 5), although the NS is highly elongated at the merger. Rather, most of the material of the NS falls into the $\mathrm{BH}$ in a short time scale $\sim 200 M_{0}$ (see the third panel of Fig. 5). A tiny fraction of the material still spreads outward during the merger phase (see the fourth panel of Fig. 5), but specific angular momentum for such material is not large enough to escape the capture by the $\mathrm{BH}$. In this case, more than $99.999 \%$ 
FIG. 5: The same as Fig. 4 but for model M40.145.

FIG. 6: The same as Fig. 4 but for model M50.145. 
of the material is eventually swallowed by the BH (cf. Fig. (7).

For model M50.145, even the mass shedding does not occur in the inspiral phase, as indicated in Fig. 2. During the merger, the NS is deformed by the tidal field of its companion $\mathrm{BH}$ (see the first and second panels of Fig. 6), but spiral arm is not formed nor angular momentum transport work. As a result, nearly all the materials are swallowed by the $\mathrm{BH}$ in a short time scale of $\lesssim 100 M_{0}$ (see the third and fourth panels of Fig. 6), and the final outcome is a rotating $\mathrm{BH}$ approximately in a vacuum spacetime.

To clarify the infall process of the material into the companion $\mathrm{BH}$, we plot evolution of the rest mass of the material located outside the apparent horizon, $M_{r>r_{\mathrm{AH}}}$, for several models in Fig. 7. Here, $M_{r>r_{\mathrm{AH}}}$ is defined by

$$
M_{r>r_{\mathrm{AH}}} \equiv \int_{r>r_{\mathrm{AH}}} \rho_{*} d^{3} x,
$$

and $r_{\mathrm{AH}}(\theta, \varphi)$ denotes the radius of the apparent horizon for given angular coordinates.

Figure 7(a) plots $M_{r>r_{\mathrm{AH}}}$ as a function of $t-t_{\text {merge, }}$, where $t_{\text {merge }}$ denotes the approximate onset time of the merger, for models M15.145, M20.145, M30.145, M40.145, and M50.145. This shows that (i) for $Q \leq 2$, a disk of rest mass $\sim 0.01-0.02 M_{*}$ is formed and the lifetime of the disk is much longer than the dynamical time scale; (ii) for $Q \geq 3$, approximately all the materials are swallowed by the $\mathrm{BH}$ in $\sim 500 M_{0}$ (the rest mass of the material located outside the apparent horizon is less than $10^{-5} M_{*}$ at the final stage; see also Table III). The time scale for the infalling is shorter for the larger values of $Q$. These facts hold irrespective of the initial orbital separation and grid resolution, as long as $Q \geq 3$. Remember the fact that the compactness of the NSs for these models is 0.145 , which is a relatively small value; the typical compactness for the NS of mass $1.3-1.4 M_{\odot}$ is between $\sim 0.14$ and $\sim 0.21$ according to theories of high-density matter [42]. Because the less compact NS (i.e., the NS of larger radius) is more subject to disk formation, we conclude that the disk (or torus) mass around a $\mathrm{BH}$ formed after the merger is negligible, for the mass ratio $Q \geq 3$. By contrast, if the mass ratio is smaller than $\sim 2$ and the compactness of the NS is relatively small as $\mathcal{C}=0.145$, a disk of mass $\sim 0.01-0.02 M_{*}$ may be formed. (We note that in the presence of $\mathrm{BH}$ spin, this conclusion changes; work in progress in our group.)

To show the further evidence that the disk is really formed for $Q=2$ and $\mathcal{C}=0.145$, we generate Fig. 7 (b). In this figure, we plot $M_{r>r_{\mathrm{AH}}}$ as a function of time for model M20.145, for model M20.145N for which the initial orbital separation is smaller than that of model M20.145, and for model M20.145b for which the initial condition is computed in the $\beta^{\varphi}$ condition. This figure shows that the disk mass at $t-t_{\text {merger }} \approx 1000 M_{0}$ is $\sim 0.01 M_{*}$ irrespective of the initial conditions for a given grid resolution $(N=36)$. This fact indicates that the initial orbital separation is large enough to exclude spurious ef- fects associated with noncircularity of the initial condition to the resulting disk mass. We note that in the previous early-stage works [14, 15], the simulations were performed from the initial conditions of small orbital separations, and consequently, it was found that the disk mass depends strongly on the initial separation, failing to draw the definitive conclusion about the disk mass. This drawback is overcome in this work.

To show dependence of $M_{r}>r_{\mathrm{AH}}$ as a function of $t$ on the compactness of the NSs, in Fig. 7)(c), we compare the results for models M20.145, M20.160, and M20.178, and for models M30.145, M30.160, and M30.178. As this figure shows, the disk mass systematically and steeply decreases with the increase of the compactness $\mathcal{C}$ for $Q=2$; the disk mass for model M20.160 is by a factor of $\sim 15$ smaller than that for model M20.145. This dependence is simply caused by the fact that the tidal disruption of more compact NSs occurs for an orbit closer to the ISCO, suppressing disk formation. This result implies that even for a small mass ratio $Q=2$, disks are not formed if the radius of the NS is not very large. Note that for a hypothetical mass of $M_{\mathrm{NS}}=1.35 M_{\odot}$ for model M20.160, the circumferential radius of the NS is $R_{\mathrm{NS}}=12.5 \mathrm{~km}$, which is not a large value because nuclear theories predict it in the range $\sim 10-15 \mathrm{~km}$ [42]. Nevertheless, the disk is not formed. This implies that for a typical NS of $M_{0}=1.35 M_{\odot}$ and $R_{\mathrm{NS}}=11-12 \mathrm{~km}$, the disk is formed only for a highly restricted case, $Q<2$, i.e., $M_{\mathrm{BH}}<2.7 M_{\odot}$. This conclusion agrees with a conjecture by Miller [43], and is also consistent with the results by Duez et al. [17] in which they show that the disk mass is at most $\sim 0.01 M_{*}$ for a compact $\mathrm{NS}$ of $\mathcal{C}=0.174$ with the most optimistic mass ratio $Q=1$. The present result also suggests that most of $\mathrm{BH}-\mathrm{NS}$ binaries may not be a promising candidate for the central engine of SGRBs [11, 12], unless the radius of the NS is fairly large $\gtrsim 14$ $\mathrm{km}$ or the $\mathrm{BH}$ has a spin.

Figure 7(c) also compares $M_{r>r_{\mathrm{AH}}}$ as a function of $t$ for models M30.145, M30.160, and M30.178. For all these cases, approximately all the materials of the NS eventually fall into the $\mathrm{BH}$ in $\sim 500 M_{0}$ after the onset of the merger. However, the merger process and subsequent infalling process into the $\mathrm{BH}$ depend strongly on the compactness of the NSs. For model M30.145, the mass-shedding and subsequent tidal disruption occur before the binary reaches the ISCO, as in model M20.145 (see also Fig. 18 of Ref. [16] for which essentially the same result as for model M30.145 is shown). As a result, a spiral arm is formed around the companion $\mathrm{BH}$ and a fraction of the material spreads outward. Then, a disk of rest mass $\gtrsim 0.1 M_{*}$ surrounding the $\mathrm{BH}$ is transiently formed, although a large fraction of the material is swallowed by the $\mathrm{BH}$ in $\sim 50 M_{0}$ after the onset of the merger. Subsequently, the material gradually accretes from the disk into the $\mathrm{BH}$ in the time duration of $\sim 150 M_{0}$. During this phase, the disk mass decreases from $\sim 0.1 M_{*}$ to $\sim 0.02 M_{*}$, and thus, for the first $\sim 150 M_{0}$ after its formation, a massive disk is present. However, the disk 

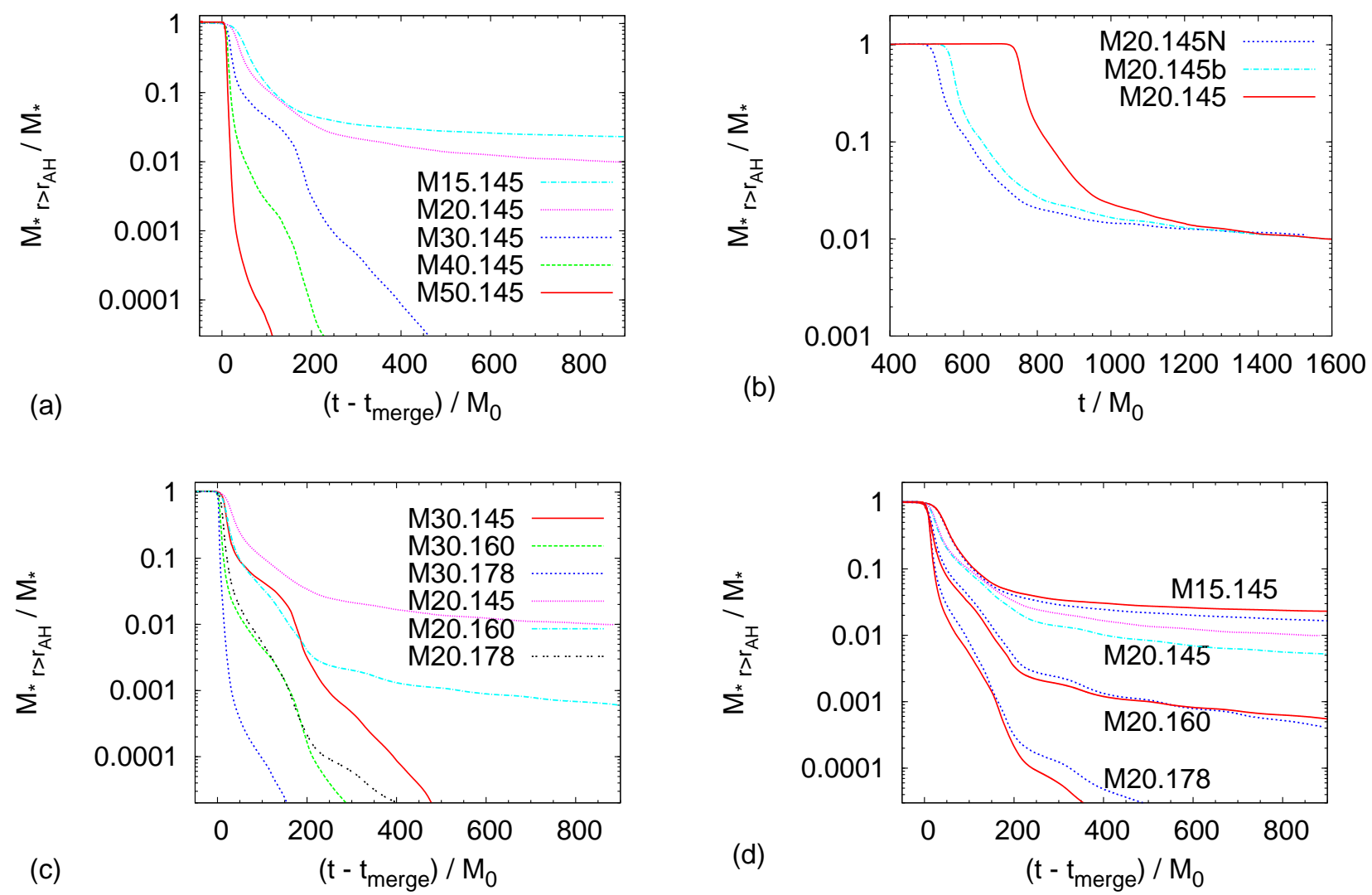

FIG. 7: Evolution of the rest mass of the material located outside the apparent horizon (a) for models M15.145, M20.145, M30.145, M40.145, and M50.145 with $N=36$, (b) for model M20.145, M20.145N, and M20.145b with $N=36$, (c) for models M20.145, M20.160, M20.178, M30.145, M30.160, and M30.178 with $N=36$, and (d) for models M15.145, M20.145, M20.160, and M20.178 with $N=36$ (solid curves) and $N=30$ (dotted curves). For a hypothetical value of $M_{\mathrm{NS}}=1.35 M_{\odot}$, $100 M_{0} \approx 1.66,2.00,2.66,3.33$, and $3.99 \mathrm{~ms}$ for $Q=1.5,2,3,4$, and 5 , respectively.

material does not have sufficiently large specific angular momentum for keeping orbits around the $\mathrm{BH}$, and eventually falls into the $\mathrm{BH}$ in a runaway manner. In the end, more than $99.999 \%$ of the material is swallowed by the $\mathrm{BH}$.

For model M30.160, a disk is formed around the BH transiently. However, its mass is much smaller than that for model M30.145 because the tidal disruption occurs at an orbit close to the ISCO, as in model M40.145. For model M30.178, a disk is not formed around the BH even transiently, as in model M50.145. The reason is that the NS in this model is not tidally disrupted before the binary reaches the ISCO. For these two cases, more than $99.999 \%$ of the material is swallowed into the $\mathrm{BH}$ within a short duration of $200-300 M_{0}$.

Before closing this section, we note that the final disk mass depends very weakly on the grid resolution for models M30.145, M30.160, M30.178, M20.160, and M20.178, whereas for models M15.145 and M20.145 for which the disk mass is $\gtrsim 0.01 M_{*}$, the final disk mass increases with improving the grid resolution [see Fig. 7(d)]. This implies that (i) for the case that the massive disk is not formed, our conclusion is based on the convergent result, whereas (ii) for the case that a disk of mass $\gtrsim 0.01 M_{*}$ is formed, the results with $N=36$ should be regarded as a lower-bound for the disk mass, and the disk mass would be larger than $0.01 M_{*}$ for model M20.145 and $0.02 M_{*}$ for model M15.145. This dependence on the grid resolution results from the fact that with the poorer grid resolutions, numerical dissipation of angular momentum is larger, increasing an amount of the material which falls into the BH. However, this systematic dependence shows that the disk of mass $\gtrsim 0.01 M_{*}$ is indeed formed.

\section{B. Black hole mass and spin after merger}

Figure 8 plots $M_{\mathrm{irr}} / M_{0}, C_{p} / C_{e}$, and $C_{e} / 4 \pi M_{0}$ of a $\mathrm{BH}$ as functions of time for models M20.145 and M40.145 (similar behavior is found for other models). Here, $C_{p}$ and $C_{e}$ are polar and equatorial circumferential radii of the $\mathrm{BH}$. An irreducible mass, $M_{\mathrm{irr}}$, of the $\mathrm{BH}$ is defined by the area of apparent horizon, $A_{\mathrm{AH}}$,

$$
M_{\mathrm{irr}}=\sqrt{\frac{A_{\mathrm{AH}}}{16 \pi}} .
$$




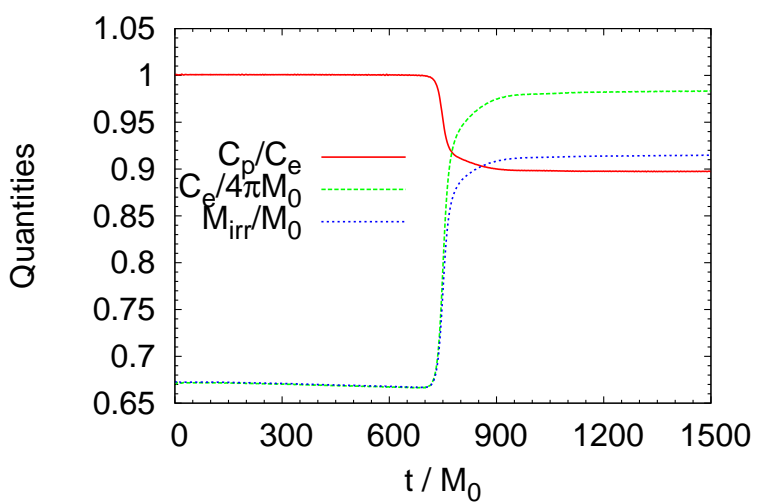

(a)

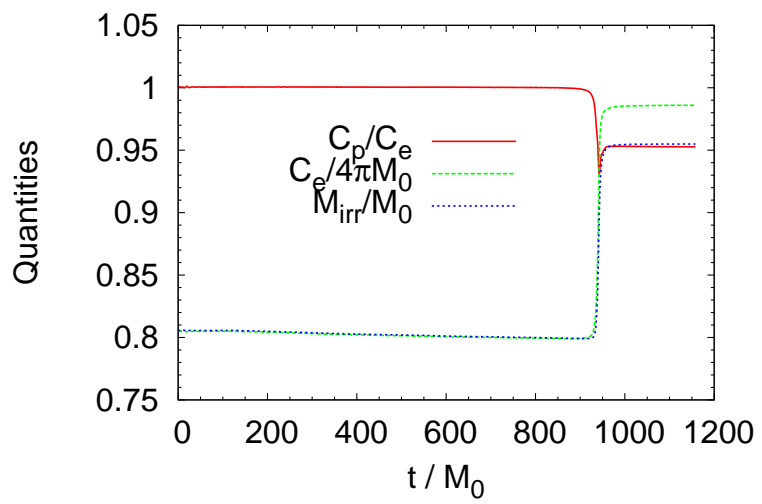

(b)

FIG. 8: Evolution of $C_{p} / C_{e}, C_{e} / 4 \pi M_{0}$, and $M_{\mathrm{irr}} / M_{0}$ as functions of time (a) for models M20.145 and (b) M40.145.

$C_{e} / 4 \pi$ is equal to the $\mathrm{BH}$ mass in stationary vacuum spacetimes of a $\mathrm{BH}$. We follow its evolution, assuming that it is approximately equal to the $\mathrm{BH}$ mass even in the dynamical spacetime.

Figure 8 shows that the values of these three quantities remain approximately constant before the onset of the merger (more specifically, before the material of the NS falls into the companion $\mathrm{BH}$ ). Because the $\mathrm{BH}$ is not spinning initially, the hypothetical "BH mass", $C_{e} / 4 \pi$, should be approximately equal to the irreducible mass $M_{\mathrm{irr}}$, and $C_{p} / C_{e}$ is approximately equal to unity. These hold except for small numerical error of magnitude $\sim 1 \%$. After the onset of tidal disruption, $C_{e} / 4 \pi$ and $M_{\text {irr }}$ quickly increase as the material of the NS falls into the $\mathrm{BH}$, and finally, they approximately reach constants. By contrast, $C_{p} / C_{e}$ decreases due to spin-up of the $\mathrm{BH}$ caused by mass accretion. Because of the presence of the $\mathrm{BH}$ spin, $C_{e} / 4 \pi$ becomes unequal to the irreducible mass after the onset of the merger.

In addition to $C_{e} / 4 \pi$, the mass of a $\mathrm{BH}$ formed after merger may be estimated approximately by evaluating the total energy dissipated by gravitational waves, $\Delta E$, and the baryon rest mass of disks surrounding the $\mathrm{BH}$ from an approximate relation of energy conservation as

$$
M_{\mathrm{BH}, \mathrm{f}} \equiv M_{0}-M_{r>r_{\mathrm{AH}}}-\Delta E .
$$

We note that in this formula, we ignore the binding energy between the $\mathrm{BH}$ and surrounding material. Thus, $M_{\mathrm{BH}, \mathrm{f}}$ is likely to give a slightly overestimated value for the true $\mathrm{BH}$ mass.

The values of $\Delta E, M_{r>r_{\mathrm{AH}}}, M_{\mathrm{BH}, \mathrm{f}}$, and $C_{e} / 4 \pi$ are listed for all the models chosen in this paper in Table III and IV. In Table III the results in the end of the simulation for $N=36$ are presented, whereas Table IV lists the results for energy and angular momentum radiated by gravitational waves. Here, the values of $\Delta E$ and $\Delta J$ depend on the radii of the wave extraction by $1-3 \%$ for $N=36$. In addition, these systematically increase with improving the grid resolution. Thus, we infer these quantities by an extrapolation of the data for $N=30$ and 36 , which carried out assuming the third-order convergence. (We note that the Einstein and hydrodynamic equations are solved in the fourth and third order schemes.)

Table III shows that $M_{\mathrm{BH}, \mathrm{f}}$ agrees with $C_{e} / 4 \pi$ within $\sim 0.5 \%$ error, but $M_{\mathrm{BH}, \mathrm{f}}$ is systematically larger than $C_{e} / 4 \pi$. The likely reason that $\Delta E$, which is used in computing $M_{\mathrm{BH}, \mathrm{f}}$, is slightly underestimated for $N=36$ because of numerical dissipation of gravitational wave amplitude: Indeed, numerical results for the energy and angular momentum radiated by gravitational waves increase with improving the grid resolution as mentioned above, and for the extrapolated results shown in Table IV another conservation relations such as

$$
\frac{C_{e}}{4 \pi}+M_{r>r_{\mathrm{AH}}}+\Delta E=M_{0}
$$

holds in a good manner within the numerical error of $\sim 0.1-0.2 \%$.

As described in Ref. [14], there are at least three methods for approximately estimating the final $\mathrm{BH}$ spin. In this paper, we use the following methods. In the first method, we approximately estimate the mass and spin of the $\mathrm{BH}$ by conservation laws. Namely, we determine them by subtracting total energy and angular momentum dissipated by gravitational waves, and rest mass and angular momentum of disks surrounding the $\mathrm{BH}$ from the initial ADM mass and angular momentum, respectively. As shown in Eq. (24), the BH mass is estimated to give $M_{\mathrm{BH}, \mathrm{f}}$. In the same manner, angular momentum of the $\mathrm{BH}$ may be estimated by

$$
J_{\mathrm{BH}, \mathrm{f}} \equiv J_{0}-J_{r>r_{\mathrm{AH}}}-\Delta J,
$$

where $\Delta J$ is total angular momentum radiated by gravitational waves and $J_{r>r_{\mathrm{AH}}}$ is angular momentum of the material located outside the apparent horizon, defined by

$$
J_{r>r_{\mathrm{AH}}} \equiv \int_{r>r_{\mathrm{AH}}} \rho_{*} h u_{\varphi} d^{3} x .
$$

Here, $u_{\varphi}=\left(x-x_{\mathrm{P}}\right) u_{y}+\left(y-y_{\mathrm{P}}\right) u_{x}$ and $\left(x_{\mathrm{P}}, y_{\mathrm{P}}\right)$ denote the position of the puncture. $J_{r>r_{\mathrm{AH}}}$ exactly gives the angular momentum of the material in the axisymmetric and stationary spacetime. In the late phase of the merger, the spacetime relaxes to a quasistationary and nearly 
axisymmetric state. Thus, we may expect that $J_{r>r_{\mathrm{AH}}}$ will provide an approximate magnitude of the angular momentum of disks. From $J_{\mathrm{BH}, \mathrm{f}}$ and $M_{\mathrm{BH}, \mathrm{f}}$, we define a nondimensional spin parameter by $a_{\mathrm{f} 1} \equiv J_{\mathrm{BH}, \mathrm{f}} / M_{\mathrm{BH}, \mathrm{f}}^{2}$ (see Table III).

In the second method, the spin is determined from the following geometric quantities of the apparent horizon; $C_{e} / 4 \pi$ and $M_{\mathrm{irr}}$. For Kerr BHs of spin $a$, the following relation holds,

$$
M_{\mathrm{irr}}=\frac{C_{e}}{4 \sqrt{2} \pi}\left(1+\sqrt{1-a^{2}}\right)^{1 / 2},
$$

and hence, $a$ is determined from $C_{e}$ and $M_{\text {irr. }}$. We estimate the spin assuming that Eq. (28) holds even for the $\mathrm{BH}$ surrounded by the disk [44]. The $\mathrm{BH}$ spin determined by this method is referred to as $a_{\mathrm{f} 2}$.

In the third method, $C_{p} / C_{e}$ is used. For Kerr BHs, it is calculated to give

$$
\frac{C_{p}}{C_{e}}=\frac{\sqrt{2 \hat{r}_{+}}}{\pi} E\left(a^{2} / 2 \hat{r}_{+}\right),
$$

where $\hat{r}_{+}=1+\sqrt{1-a^{2}}$ and $E(z)$ is an elliptic integral defined by

$$
E(z)=\int_{0}^{\pi / 2} \sqrt{1-z \sin ^{2} \theta} d \theta .
$$

Thus, assuming that the same relation holds even for the $\mathrm{BH}$ surrounded by the disk, we may estimate a $\mathrm{BH}$ spin from $C_{p} / C_{e}$. We refer to this spin as $a_{\mathrm{f} 3}$.

The values of $a_{\mathrm{f} 1}-a_{\mathrm{f} 3}$ for $N=36$ are listed in Table III. We find that three values agree within a few $\%$ for $Q=3-5$. The values of the spin depend weakly on the compactness of the NSs, and hence, we conclude that the spin parameter of the formed $\mathrm{BH}$ is $\approx 0.56 \pm 0.01,0.48 \pm$ 0.01 , and $0.42 \pm 0.01$ for $Q=3,4$, and 5, respectively. For the smaller values of $Q$, the final $\mathrm{BH}$ spin is larger. The reason for this is that for the larger mass ratio, the total angular momentum of the system $J$ at a given value of $m_{0} \Omega$ in the inspiral orbit is approximately proportional to $m_{0}^{2} Q /(1+Q)^{2}$ (see also the initial condition in Table I). Thus, the binaries of smaller values of $Q$ should form a $\mathrm{BH}$ of higher spin.

For the case that $Q \leq 2$ and the disk mass is $\gtrsim 0.01 M_{*}$, $a_{\mathrm{f} 1}$ does not agree well with $a_{\mathrm{f} 2}$ and $a_{\mathrm{f} 3}$ with the error size $\sim 0.05$. Because $a_{\mathrm{f} 2}$ and $a_{\mathrm{f} 3}$ agree well, the error in $a_{\mathrm{f} 1}$ seems to be much larger than those of $a_{\mathrm{f} 2}$ and $a_{\mathrm{f} 3}$. Then, the possible error sources are (i) underestimation of angular momentum that the disks possess and/or (ii) underestimation of angular momentum dissipated by gravitational waves. The possibility (ii) is not very likely, because for $Q \geq 3$ and for models M20.160 and M20.178, $a_{\mathrm{f} 1}-a_{\mathrm{f} 3}$ agree in a much better manner, indicating that gravitational waves are computed with a good accuracy. A possible reason that the angular momentum of the disk is underestimated is that many of the disk materials are located not in the finest grid domain in the AMR grid but in the second-fourth finest domains in which the grid resolution may not be high enough, and hence, the angular momentum is spuriously dissipated. This reason is also inferred from Fig. 7 (d), which shows that the disk mass surrounding $\mathrm{BH}$ depends on the grid resolution.

\section{Gravitational waves}

\section{Comparison with Taylor T4 waveform}

Figure9(a)-(f) plot gravitational waveforms (+ mode) observed along the $z$ axis as a function of the retarded time for models M20.145-M50.145, M20.160, and M30.178. ( $h$ denotes a gravitational wave amplitude.) The retarded time is approximately defined by

$$
t_{\text {ret }} \equiv t-D-2 M_{0} \ln \left(D / M_{0}\right) \text {, }
$$

where $D$ is a distance between the source and an observer. The gravitational waveforms shown here are obtained by performing the time integration of the Newman-Penrose quantity of $l=|m|=2$ mode. From the values of $D h / m_{0}$ shown in Fig. 9, the amplitude of gravitational waves at a distance $D$ is evaluated by

$$
\begin{aligned}
h_{\mathrm{gw}} \approx 2.4 \times 10^{-} & 22\left(\frac{D h / m_{0}}{0.1}\right) \\
& \times\left(\frac{100 \mathrm{Mpc}}{D}\right)\left(\frac{m_{0}}{5 M_{\odot}}\right) .
\end{aligned}
$$

To validate the numerical waveforms presented here, we first compare the waveforms in the inspiral phase with those derived by the so-called Taylor-T4 formula for two point masses in quasicircular orbits. In the Taylor-T4 formula, one calculates evolution of the angular velocity, $\Omega$, of the quasicircular orbits due to the gravitational radiation reaction up to the $3.5 \mathrm{PN}$ level beyond the quadrupole formula: The circular orbits at a given value of $\Omega$ are determined by the 3PN equations of motion neglecting gravitational radiation reaction, and then, one considers an adiabatic evolution of $\Omega$ using the $3.5 \mathrm{PN}$ formula for gravitational radiation reaction (see, e.g., Refs. [45, 46] for a detailed description of various formulas based on the PN theory). Recent high-accuracy simulations for equal-mass (nonspinning or corotating) $\mathrm{BH}-\mathrm{BH}$ binaries have proven that the Taylor-T4 formula provides their orbital evolution and gravitational waveforms with a high accuracy at least up to about one orbit before the onset of the merger. Assuming that this holds for unequal-mass binaries, we calibrate our numerical results by comparing them with the results by the Taylor$\mathrm{T} 4$ formula. (Indeed, our numerical results indicate that the Taylor-T4 formula provides a good approximate solution for $\Omega(t)$ even for the nonequal-mass binaries as shown below.) In the present work, the 3PN formula [47] is employed for calculating the amplitude of gravitational waves in the Taylor-T4 formula. 
TABLE III: Rest mass of material located outside apparent horizon $\left(M_{r>r_{\mathrm{AH}}}\right)$, BH mass estimated by energy-conservation law $\left(M_{\mathrm{BH}, \mathrm{f}}\right)$, BH mass estimated from equatorial circumferential radius $\left(C_{e} / 4 \pi\right)$, irreducible mass of the $\mathrm{BH}\left(M_{\mathrm{irr}}\right.$; square root of area of apparent horizon in units of $16 \pi)$, ratio of polar circumferential radius $\left(C_{p}\right)$ to equatorial one $\left(C_{e}\right)$ of apparent horizon (i.e., $C_{p} / C_{e}$ ), and estimated spin parameters of the final state of the BH. $a_{\mathrm{f} 1}, a_{\mathrm{f} 2}$, and $a_{\mathrm{f} 3}$ are computed from BH mass and angular momentum estimated by conservation laws, from $M_{\mathrm{irr}}$ and $C_{e}$ of apparent horizon, and from $C_{p} / C_{e}$, respectively. All the values presented here are measured for the state obtained at the end of the simulations for $N=36$. Note that the parameters for the BH still vary with time at the end of the simulation for models M15.145, M20.145, and M20.145N because of gradual mass accretion. The error for $C_{e}$ and $C_{p} / C_{e}$ is $\lesssim 0.1 \%$, whereas that for $a_{\mathrm{f} 1}, a_{\mathrm{f} 2}$, and $a_{\mathrm{f} 3}$ is $\lesssim 0.01$ except for the case that the disk is formed for which the error of $a_{\mathrm{f} 1}$ would be $\sim 0.05$. The values for the final state of the BH depend very weakly on the grid resolution as far as $N \geq 30$, but the mass of disk which presents only for $Q \leq 2$ systematically increases with $N$. The present results should be regarded as the lower-bound for the disk mass.

\begin{tabular}{ccccccccc}
\hline Model & $M_{r>r_{\mathrm{AH}}} / M_{*}$ & $M_{\mathrm{BH}, \mathrm{f}} / M_{0}$ & $C_{e} / 4 \pi M_{0}$ & $M_{\mathrm{irr}} / M_{0}$ & $C_{p} / C_{e}$ & $a_{\mathrm{f} 1}$ & $a_{\mathrm{f} 2}$ & $a_{\mathrm{f} 3}$ \\
\hline M15.145 & 0.023 & 0.983 & 0.981 & 0.895 & 0.872 & 0.801 & 0.747 & 0.750 \\
\hline M20.145 & 0.010 & 0.988 & 0.984 & 0.915 & 0.898 & 0.717 & 0.684 & 0.682 \\
\hline M20.145N & 0.011 & 0.988 & 0.983 & 0.916 & 0.900 & 0.721 & 0.677 & 0.676 \\
\hline M20.160 & $6 \times 10^{-4}$ & 0.988 & 0.987 & 0.919 & 0.899 & 0.694 & 0.679 & 0.680 \\
\hline M20.178 & $<10^{-5}$ & 0.983 & 0.983 & 0.917 & 0.904 & 0.676 & 0.672 & 0.666 \\
\hline M30.145 & $<10^{-5}$ & 0.989 & 0.985 & 0.942 & 0.934 & 0.566 & 0.559 & 0.564 \\
\hline M30.160 & $<10^{-5}$ & 0.985 & 0.983 & 0.940 & 0.935 & 0.547 & 0.560 & 0.562 \\
\hline M30.178 & $<10^{-5}$ & 0.982 & 0.981 & 0.940 & 0.937 & 0.551 & 0.550 & 0.552 \\
\hline M40.145 & $<10^{-5}$ & 0.988 & 0.986 & 0.955 & 0.953 & 0.485 & 0.482 & 0.474 \\
\hline M50.145 & $<10^{-5}$ & 0.989 & 0.986 & 0.963 & 0.964 & 0.408 & 0.419 & 0.425 \\
\hline
\end{tabular}

TABLE IV: Several outputs of gravitational waves. Total radiated energy $(\Delta E)$ and angular momentum $(\Delta J)$ in units of initial ADM mass $\left(M_{0}\right)$ and initial angular momentum $\left(J_{0}\right)$, fraction of radiated energy for $(l,|m|)=(2,2),(3,3),(4,4)$, and $(2,1)$ modes, frequency of the fundamental quasinormal mode, kick velocity, and type of the gravitational waveform. The radiated energy for each mode is shown in unit of $M_{0}$ by \%. The origin of the error bar is primarily the numerical error associated with the finite grid resolution, and in part, the finite extraction radii of gravitational waves. Note that gravitational waves are extracted for several coordinate radii of $50-100 M_{0}$.

\begin{tabular}{cccccccccc}
\hline Model & $\Delta E / M_{0}(\%)$ & $\Delta J / J_{0}(\%)$ & $(2,2)$ & $(3,3)$ & $(4,4)$ & $(2,1)$ & $f_{\mathrm{QNM}} M_{\mathrm{BH}} V_{\mathrm{kick}}(\mathrm{km})$ & Type \\
\hline M15.145 & $0.68 \pm 0.02$ & $14 \pm 1$ & $0.66 \pm 0.02$ & $0.006 \pm 0.002$ & $0.003 \pm 0.001$ & $\lesssim 0.01$ & - & $<5$ \\
\hline M20.145 & $0.87 \pm 0.02$ & $17.4 \pm 0.3$ & $0.85 \pm 0.01$ & $0.017 \pm 0.002$ & $0.005 \pm 0.001$ & $0.003 \pm 0.001$ & - & $21 \pm 5$ & $\mathrm{I}$ \\
\hline M20.145N & $0.78 \pm 0.02$ & $15.0 \pm 0.2$ & $0.76 \pm 0.01$ & $0.014 \pm 0.001$ & $0.005 \pm 0.001$ & $0.002 \pm 0.001$ & - & $11 \pm 2$ & I \\
\hline M20.160 & $1.22 \pm 0.02$ & $22 \pm 1$ & $1.19 \pm 0.02$ & $0.025 \pm 0.005$ & $0.006 \pm 0.002$ & $\lesssim 0.01$ & - & $62 \pm 15$ & II \\
\hline M20.178 & $1.7 \pm 0.1$ & $25 \pm 1$ & $1.6 \pm 0.1$ & $0.05 \pm 0.01$ & $0.01 \pm 0.005$ & $0.03 \pm 0.01$ & 0.087 & $126 \pm 20$ & II \\
\hline M30.145 & $1.3 \pm 0.1$ & $22.0 \pm 0.4$ & $1.2 \pm 0.1$ & $0.08 \pm 0.01$ & $0.014 \pm 0.002$ & $0.03 \pm 0.02$ & 0.081 & $98 \pm 9$ & II \\
\hline M30.160 & $1.6 \pm 0.1$ & $26 \pm 1$ & $1.4 \pm 0.1$ & $0.09 \pm 0.02$ & $0.020 \pm 0.003$ & $0.05 \pm 0.02$ & 0.080 & $153 \pm 16$ & III \\
\hline M30.178 & $1.8 \pm 0.1$ & $26 \pm 1$ & $1.7 \pm 0.1$ & $0.12 \pm 0.01$ & $0.02 \pm 0.01$ & $0.04 \pm 0.03$ & 0.080 & $137 \pm 43$ & III \\
\hline M40.145 & $1.3 \pm 0.1$ & $23.5 \pm 0.5$ & $1.1 \pm 0.1$ & $0.13 \pm 0.01$ & $0.025 \pm 0.005$ & $0.06 \pm 0.02$ & 0.077 & $136 \pm 12$ & III \\
\hline M50.145 & $1.11 \pm 0.05$ & $24 \pm 1$ & $0.85 \pm 0.02$ & $0.13 \pm 0.01$ & $0.04 \pm 0.01$ & $0.09 \pm 0.01$ & 0.074 & $137 \pm 6$ & III \\
\hline
\end{tabular}

More specifically, the comparison of the numerical waveforms and semianalytic ones derived by the TaylorT4 formula is carried out in the following manner. First, we derive the orbital angular velocity as a function of time for a numerical result from $\Psi_{4}$ by [45]

$$
\Omega(t)=\frac{1}{2} \frac{\left|\Psi_{4}(l=m=2)\right|}{\left|\int d t \Psi_{4}(l=m=2)\right|},
$$

where $\Psi_{4}(l=m=2)$ is the $l=m=2$ mode of $\Psi_{4}$. Then, we compare the numerical result for $\Omega(t)$ with the semianalytic one derived by the Taylor-T4 formula (see
Fig. (10). When comparing two results, we have a degree of freedom for the time translation. Thus, first of all, by shifting the time axis of the Taylor-T4's result, we align the origin of the time. As shown in Fig. 10, we can always shift the time axis appropriately to align two results for $\Omega(t)$.

After the appropriate time translation, we compare the gravitational waveforms obtained by the numerical simulation and by the Taylor-T4 formula. Then, we still have a degree of freedom for choosing the wave phase. Thus, we iteratively change the phase of the Taylor-T4's waveform until a good matching of two waveforms is achieved.

In Fig. 9, the dot-dotted curve denote the resulting 

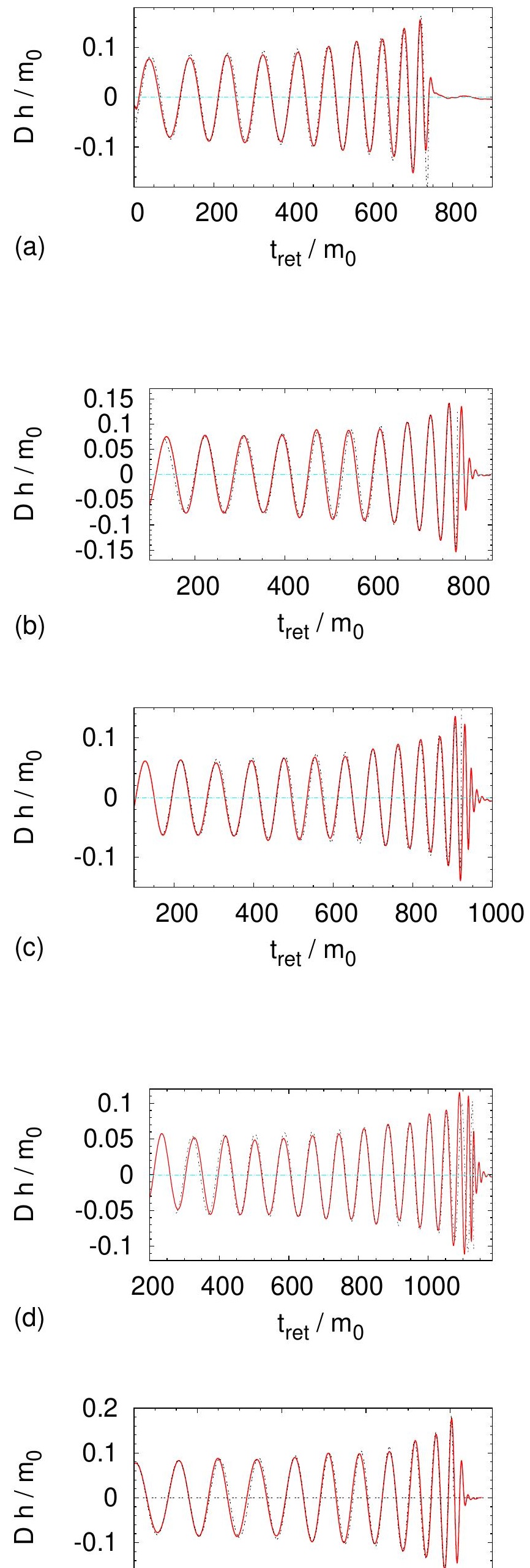

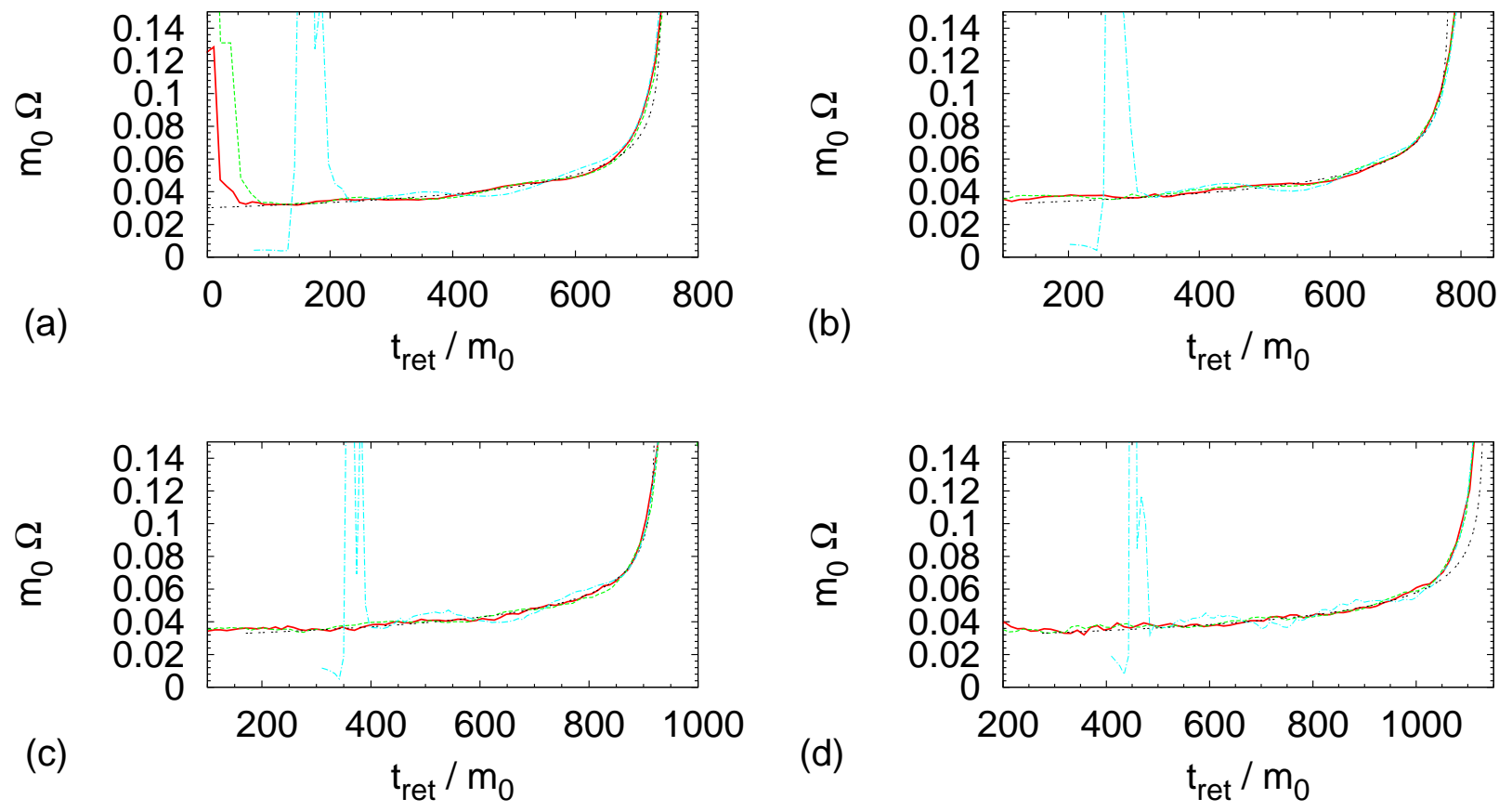

FIG. 10: Orbital angular velocity computed from $\Psi_{4}$ as a function of the retarded time for models (a) M20.145, (b) M30.145, (c) M40.145, and (d) M50.145. $t_{\text {ret }}$ denotes the retarded time and $m_{0}$ is the total mass. For all the panels, the results for two different grid resolutions with $N=30$ (dashed curve) and 36 (solid curve) and the results for M20.145b-M50.145b (dot-dashed curves) are shown together. The dot-dotted curve denotes the result derived by the Taylor-T4 formula.

semianalytic waveform derived by the Taylor-T4 formula. It is found that the numerical waveforms agree with the Taylor-T4's results with a good accuracy, except for the early stage of the simulations (i.e., for the first a few wave cycles), during which the eccentricity of the binaries is not small. In particular, for the last several wave cycles (except for the orbit just before the merger), the numerical wave phases agree with those derived by the TaylorT4 formula with in $\sim 3 \%$ error. This indicates that the binaries computed in the present simulation are indeed in an approximately quasicircular orbit of small eccentricity at least for the last several inspiral orbits. Also, this indicates that the Taylor-T4 formula provides a good approximate solution for $\Omega(t)$ even for the nonequal-mass binaries, because the agreement systematically holds irrespective of the mass ratio.

Figure 10 also shows good agreement between the numerical and Taylor-T4's results for $\Omega(t)$. The numerical results for two grid resolutions $[N=36$ (solid curve) and 30 (dashed curve)] are shown for illustrating that a convergence is approximately achieved. In addition, the results for models M20.145b-M50.145b are plotted for comparison. This figure shows that the evolution of $\Omega(t)$ for models M20.145-M50.145 agrees well with those predicted by the Taylor-T4 formula for a long time duration irrespective of the mass ratio. Modulation in the evolution of $\Omega$ is $\Delta \Omega / \Omega \lesssim 6 \%$ for the last a few inspiral phase (irrespective of $N=30$ or 36), and thus, the eccentricity, which is approximately estimated by $2 \Delta \Omega / 3 \Omega$, is $\lesssim 4 \%$. In the last several orbits, the eccentricity appears to be at most $\sim 1 \%$.

Comparing the results of M20.145-M50.145 with those of M20.145b-M50.145b, we find that the modulation is by a factor of $\gtrsim 2$ larger with the initial condition computed in the $\beta^{\varphi}$ condition. This unfavored behavior is more significant for the larger values of $Q$. This demonstrates the advantage for using the initial condition computed in the $3 \mathrm{PN}-\mathrm{J}$ condition.

\section{Classification of waveforms}

Figure 9 shows that gravitational waveforms in the merger and ringdown phases depend sensitively on the mass ratio and compactness of the NS. Comparison of the waveforms for models M20.145 and M20.160 [see Fig. 9 (a) and (e)], for which masses of the $\mathrm{BH}$ and NS are identical each other, illustrates a strong dependence of the merger waveforms on the NS compactness. The wave amplitude for model M20.145 decreases suddenly in the middle of the inspiral phase due to tidal disruption. By contrast, the wave amplitude for model M20.160 does not decrease as quickly as that for model M20.145 because the tidal disruption does not occur far outside the ISCO.

Gravitational waveforms in the merger and ringdown phases for models M30.145 and M30.178 [see Fig. 9 (b) and (f), and Fig. [11(d)] are also distinguishable because the amplitude in these phases is much larger for model M30.178. This is due to the fact that the NS for model 

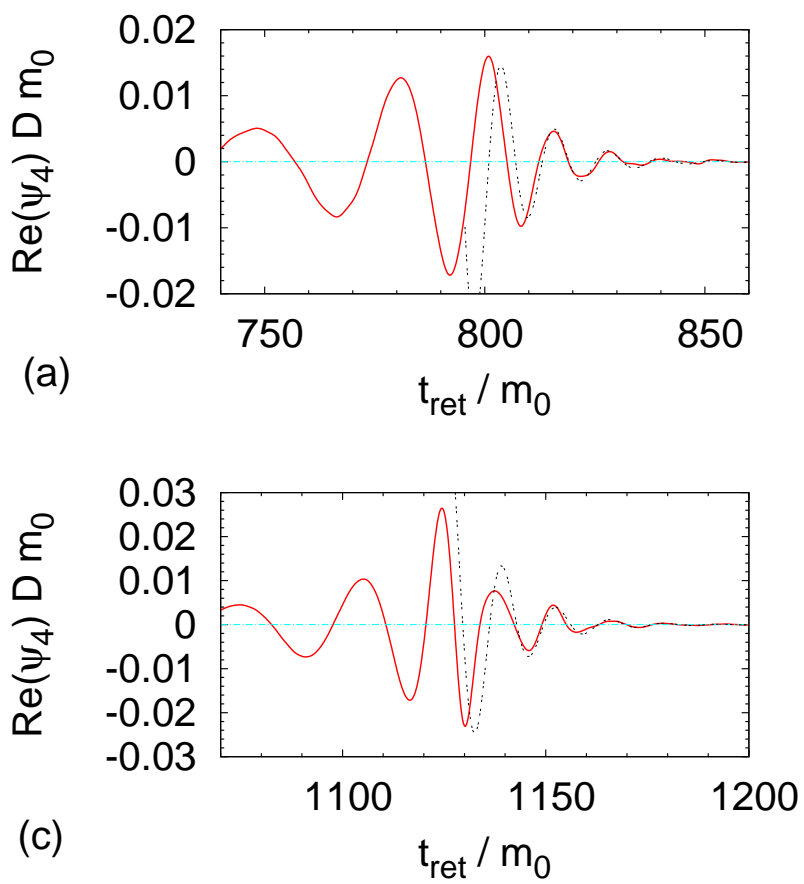
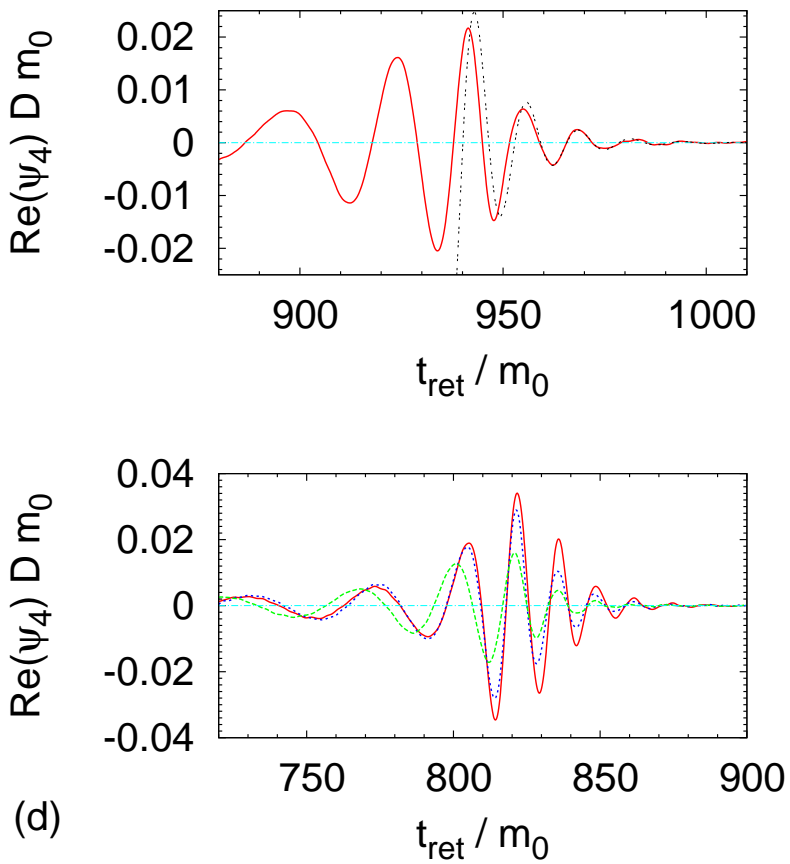

FIG. 11: Gravitational waves (the real part of $\Psi_{4}$ ) emitted in the merger and ringdown phases for models (a) M30.145, (b) M40.145, (c) M50.145, and (d) M30.145 (dashed curve), M30.160 (dotted curve), and M30.178 (solid curve). For (a)-(c), a fitting waveform given by Eq. (34) is plotted together by the dot-dotted curve.

M30.178 is not strongly affected by the tidal force of the companion $\mathrm{BH}$ even at the ISCO. By contrast, the tidal effects play an important role for deformation of the NS in close orbits for model M30.145. Because the NS is disrupted near the ISCO for this model, the wave amplitude in the merger and ringdown phases is significantly suppressed. These results illustrate that gravitational waves emitted in the merger and ringdown phases have a potential information about the compactness of the NS (see also Sec. IVD).

As these comparisons clarify, there are three types of gravitational waveforms. For the case that the NS is tidally disrupted during the inspiral phase (e.g., for model M20.145), the wave amplitude quickly decreases at the tidal disruption, as the waveforms associated with the inspiral motion are suddenly shut off. Namely, the waveform is composed only of the inspiral waveform and subsequent sudden shut-off, and the merger and ringdown waveforms are essentially absent. We refer to this type as the type I.

Even in this case, most of the material of the tidally disrupted NS falls into the companion BH (see Fig. 7). During the tidal disruption and subsequent infalling into the $\mathrm{BH}$, an orbital motion of the disrupted material and an oscillation of the BH may excite merger and ringdown gravitational waves (here "ringdown gravitational waves" imply gravitational waves associated with quasinormal modes of the BH). However, such waveforms are not seen. The likely reason for the absence of the merger waveform is that the NS is significantly elongated in a short time scale and its density quickly decreases, suppressing an ef- ficient excitation of gravitational waves. The reason for the absence of the ringdown waveform is that the material of the NS, which falls into the $\mathrm{BH}$, does not have a compact configuration but have an elongated low-density configuration. In the case that such low-density diffuse matter incoherently falls into the $\mathrm{BH}$, the excitation of the quasinormal modes is significantly suppressed due to the phase cancellation effect [48].

For models M30.145 and M20.160, mass shedding occurs before the binaries reach the ISCO. However, the sudden shut-off of the inspiral waveforms is not seen because the tidal disruption and the subsequent spreading of the material do not occur during the inspiral phase. Rather, most part of the elongated NS falls into the $\mathrm{BH}$ before the tidal disruption is completed. In this case, the ringdown waveform is seen but the amplitude is low because the quasinormal mode is not excited efficiently. By contrast, just before the ringdown gravitational waves are emitted, gravitational waves are significantly excited by a matter motion around the $\mathrm{BH}$. Thus, the merger gravitational waves are present. Namely, in these cases, the gravitational waveforms are composed primarily of the inspiral and merger ones. We refer to this type as the type II.

For models M40.145, M50.145, and M30.178, tidal effects to the NS do not play an important role. In this case, the gravitational waveform is composed of the inspiral, merger, and ringdown waveforms, as in the merger of BH-BH binaries (e.g., [45, 46]). We refer to this type as the type III. 


\section{Ringdown waveforms}

For models M30.145, M40.145, M50.145, M30.160, M20.178, and M30.178, ringdown gravitational waves associated with quasinormal modes of the formed $\mathrm{BH}$ are excited in the final phase. These gravitational waves are approximately described by

$$
A e^{-t / t_{d}} \sin \left(2 \pi f_{\mathrm{QNM}} t+\delta\right),
$$

where $A$ and $\delta$ are constants, and $f_{\mathrm{QNM}}$ and $t_{d}$ are the frequency and damping time scale of the fundamental quasinormal mode. A perturbation study predicts the frequency and damping time scale for a $\mathrm{BH}$ of mass $M_{\mathrm{BH}}$ and spin $a$ as [49]

$$
\begin{aligned}
& f_{\mathrm{QNM}} M_{\mathrm{BH}} \approx 0.16\left[1-0.63(1-a)^{0.3}\right], \\
& t_{d} \approx \frac{2(1-a)^{-0.45}}{\pi f_{\mathrm{QNM}}} .
\end{aligned}
$$

For models M30.145, M40.145, and M50.145, the BH spin is $\approx 0.56,0.48$, and 0.42 as shown in Sec. IVB. (For models M30.160 and M30.178, the spin agrees approximately with that for model M30.145.) Thus, for each of these models, $f_{\mathrm{QNM}} M_{\mathrm{BH}} \approx 0.081,0.077$, and 0.074 $\left(f_{\mathrm{QNM}} M_{0} \approx 0.082,0.078\right.$, and 0.075$)$, and $\pi t_{d} f_{\mathrm{QNM}} \approx$ $2.9,2.7$, and 2.6 , respectively.

Figure 11(a)-(c) compares the numerical waveforms in the ringdown phase with the hypothetical analytic waveforms for models M30.145-M50.145. This shows that the numerical waveforms are fitted by the analytic one (34) fairly well, and that $f_{\mathrm{QNM}}$ and $t_{d}$ computed from the data of the BH geometry agree approximately with those computed from gravitational waveforms. However, the numerical waveforms do not agree completely with the hypothetical ones. This disagreement is reasonable because for these models, the material of the NS does not simultaneously fall into the $\mathrm{BH}$ at the merger. Thus, gravitational waves are emitted both by a motion of the material moving in the vicinity of the $\mathrm{BH}$ and by the quasinormal-mode oscillation of the $\mathrm{BH}$. In addition, the quasinormal modes are not simultaneously excited because the material does not simultaneously fall into the $\mathrm{BH}$, and the resulting waveforms may be composed of many ringdown waveforms, as well as of the waveforms excited by a material moving around the BH. Furthermore, the system is not completely in a vacuum nor in a stationary state, and hence, the numerical waveforms may not be fitted precisely by the analytic results derived in the ideal assumption.

The wavelength of gravitational waves emitted in the merger phase (around the time when the peak amplitude is reached) is slightly longer than that of the quasinormal mode (i.e., the frequency is lower than $f_{\mathrm{QNM}}$ ). This indicates that these gravitational waves are not emitted by an oscillation of the BH, but they are likely to be emitted primarily by a motion of the material which moves in the vicinity of the $\mathrm{BH}$. In the merger phase, the amplitude gradually decreases after the peak is reached. The reason for this behavior is that the material is elongated by the tidal effect of the $\mathrm{BH}$ during the infalling; i.e., the reason is not the damping associated with the quasinormal mode oscillation. The amplitude emitted in the merger phase is much larger than that emitted in the ringdown phase, although the characteristic frequencies of these two types of gravitational waves are not very different. In Sec. IVD, we find that the Fourier spectrum has a plateau in a high frequency region $f m_{0} \sim 0.06-0.08$ for the case that $Q=3-5$. This plateau is primarily generated by gravitational waves emitted in the merger phase not by the quasinormal mode oscillation.

Figure 11 (d) compares the waveforms emitted in the merger and ringdown phases for models M30.145, M30.160, and M30.178. For these models, the masses of the BH and NS are identical each other, but the compactness of the NSs is different. This figure shows that the amplitude is larger for the model with the larger value of $\mathcal{C}$. This is reasonable because more compact NSs are less subject to the tidal effects by the companion $\mathrm{BH}$, and hence, the material of the NS falls into the $\mathrm{BH}$ in more simultaneous manner, resulting in a coherent excitation of gravitational waves.

The difference in the amplitude of gravitational waves emitted in the merger and ringdown phases is reflected also in the noticeable difference of energy and angular momentum carried by gravitational waves. As shown in Table IV, for example, the total energy radiated for models M30.160 and M30.178 is by $\sim 35 \%$ and $\sim 50 \%$ larger than that for model M30.145, respectively.

\section{Gravitational wave spectrum}

To determine the effective amplitude of gravitational waves for a given frequency, the Fourier spectrum of gravitational waves of $l=|m|=2$ modes are computed. In this paper, as the Fourier spectrum, we define

$$
h(f) \equiv \sqrt{\frac{\left|h_{+}(f)\right|^{2}+\left|h_{\times}(f)\right|^{2}}{2}},
$$

where $f$ is the frequency, $h_{+}(f)$ and $h_{\times}(f)$ are the Fourier transformation of the plus and cross modes of gravitational waves observed along the $z$ axis;

$$
\begin{aligned}
& h_{+}(f)=\int e^{2 \pi i f t} h_{+}(t) d t, \\
& h_{\times}(f)=\int e^{2 \pi i f t} h_{\times}(t) d t .
\end{aligned}
$$

Then, the most optimistic effective amplitude of gravitational waves for a given frequency is defined by $f h(f)$.

In the numerical simulations, BH-NS binaries of a finite orbital separation are prepared as the initial condition and the inspiral phase is computed for a finite duration. Consequently, the Fourier spectrum for the low-frequency side (for $f \lesssim \Omega_{0} / \pi$ ) becomes absent if we 

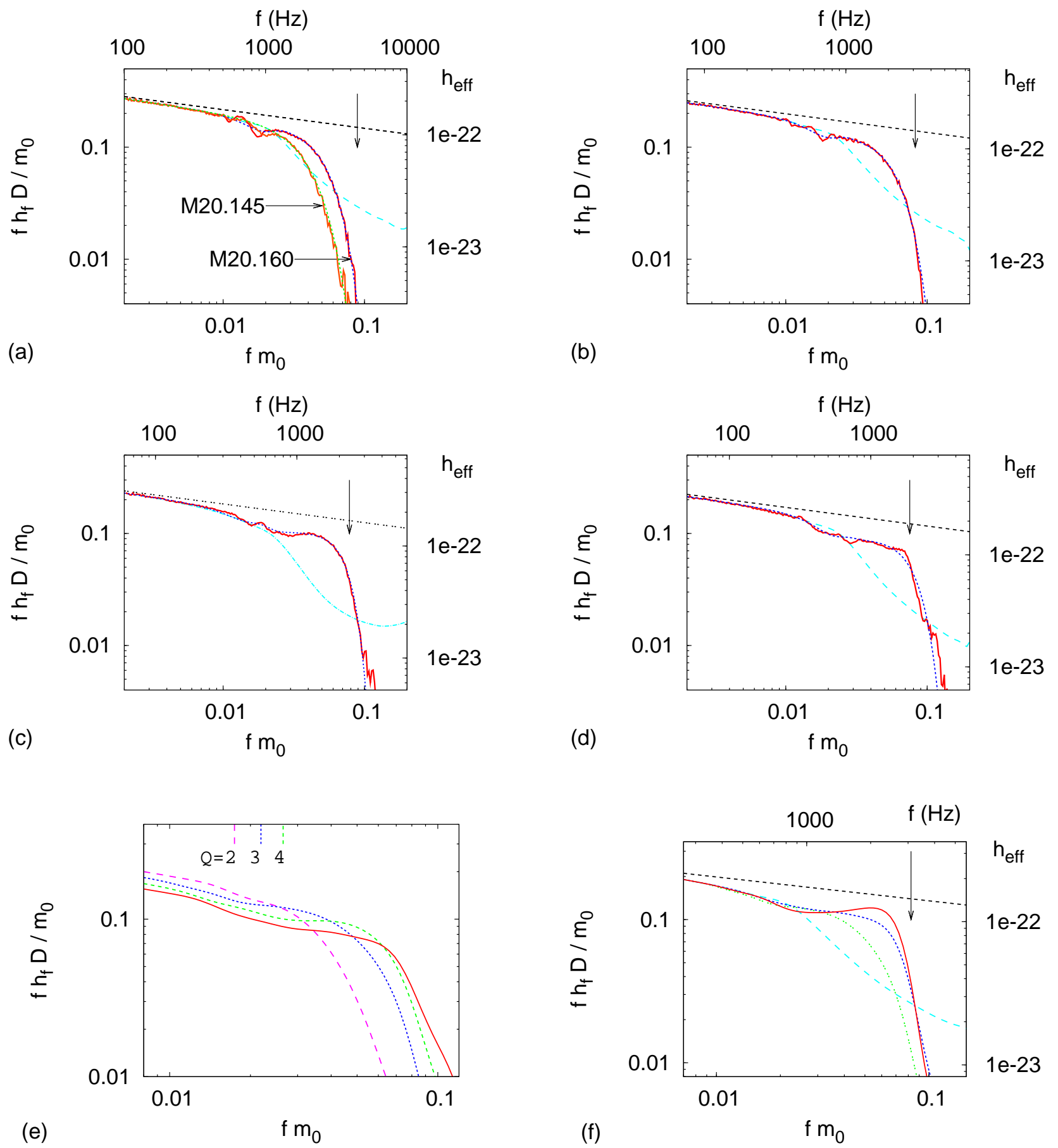

(e)

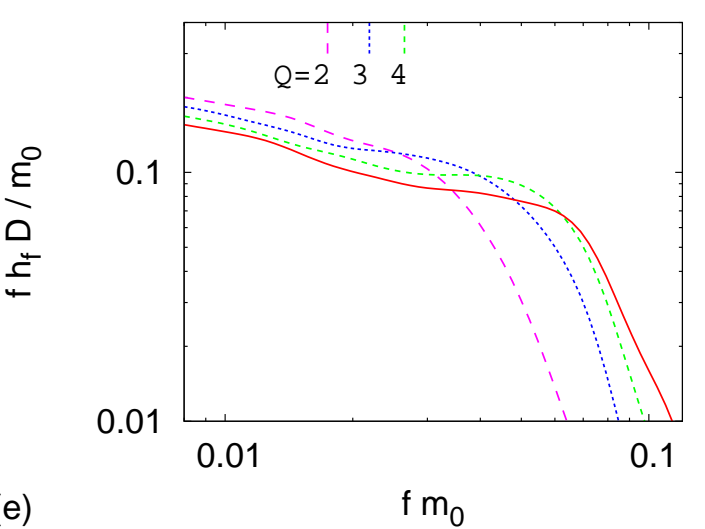

FIG. 12: (a)-(d) The spectrum of gravitational waves $f h(f) D / m_{0}$ (solid curves) for models (a) M20.145 and M20.160, (b) M30.145, (c) M40.145, and (d) M50.145, respectively. The dashed and long-dashed curves denote the relation according to Eq. (40) and spectra of gravitational waves computed in the Taylor-T4 formula. The dotted curves denote the results of fitting. The upper horizontal and right vertical axes show the value in a hypothetical value of $M_{\mathrm{NS}}=1.35 M_{\odot}$ and $D=100 \mathrm{Mpc}$. The arrow indicates the frequency of the fundamental quasinormal mode calculated by Eq. (36). (e) The same as (a) but for gathering the spectra for models M20.145-M50.145 (long-dashed, dotted, dashed, and solid curves). To clarify the qualitative feature of the spectra, a smoothing procedure is applied for the numerical data. The three lines in the upper left side denote the predicted frequency at which mass shedding of the NS occurs due to tidal field of the companion BH for models M20.145-M40.145 ( $Q$ denotes the mass ratio). (f) The same as (b) but for models M30.145 (dot-dotted curve), M30.160 (dotted curve), and M30.178 (solid curve). A smoothing procedure is also applied for the numerical data. 
naively perform the Fourier transformation for the numerical data. To compensate the Fourier spectrum for the low-frequency side, we combine a hypothetical waveform computed by the Taylor-T4 formula, as often done (e.g., Refs. [14, 45]). To do so, we match the numerical waveforms with those by the Taylor-T4 formula at a time when the corresponding binary orbit in the numerical simulation is approximately in a quasicircular state. As shown in Fig. 9] two waveforms match well for a wide range of time, so the resulting Fourier spectrum depends only weakly on the chosen matching time.

Figure 12 plots $f h(f) D / m_{0}$ for various models. In Fig. 12 (a)-(d), we also plot the Fourier spectra of a gravitational waveform derived in the Taylor-T4 formula (long-dashed curve) and by the Newtonian waveform (dashed curve) as (e.g., [50])

$$
f h(f)=\sqrt{\frac{5}{24 \pi}} \frac{m_{0}}{D} \frac{Q^{1 / 2}}{1+Q}\left(\pi m_{0} f\right)^{-1 / 6} .
$$

Here, "Newtonian" implies that the orbital motion is calculated in the Newtonian plus 2.5 PN equations of motion, and the gravitational waveform is computed by the quadrupole formula.

As Figure 12 indicates, the spectra of gravitational waves emitted in the inspiral, merger, and ringdown phases are smoothly connected. Nevertheless, we still see modulations of small amplitude for $0.01 \lesssim f m_{0} \lesssim 0.02$, which are likely to be caused by slight modulations in the amplitude and/or phase of numerical gravitational waveforms.

In the upper-horizontal and right-vertical axis, we plot the frequency and averaged effective amplitude for hypothetical values $M_{\mathrm{NS}}=1.35 M_{\odot}$ and $D=100 \mathrm{Mpc}$. Here, the averaged effective amplitude is defined by the average of $f h(f)$ over the source direction and the direction of the binary orbital plane as

$$
\begin{aligned}
& h_{\mathrm{eff}} \equiv 0.4 f h(f) \\
&=9.6 \times 10^{-23}\left(\frac{f h(f) D / m_{0}}{0.1}\right)\left(\frac{m_{0}}{5 M_{\odot}}\right) \\
& \quad \times\left(\frac{D}{100 \mathrm{Mpc}}\right)^{-1} .
\end{aligned}
$$

Figure 12 shows that the spectrum shape has the following universal qualitative feature: Irrespective of the values of $\mathcal{C}$ and $Q, f h(f)$ (hereafter referred to as the spectrum amplitude) decreases with the increase of $f$ and above a "cut-off" frequency, $f_{\text {cut }}$, it decreases exponentially. For $f \rightarrow 0, f h(f)$ is universally proportional to $f^{-1 / 6}$, and $f<f_{\text {cut }}, f h(f)$ is always written as $\propto f^{-1 / 6} F(f)$, where $F(f)$ is a slowly varying function of $f$ and the value is $\lesssim 1$. However, the detailed spectrum shape depends on the values of $\mathcal{C}$ and $Q$, and Fig. 12 exhibits a wide variety of the possible spectrum shape as explained in the following.

For the case that the NS is tidally disrupted outside the ISCO, type I gravitational waves are emitted (e.g., for model M20.145). In this case, the spectrum amplitude monotonically decreases, and above a cut-off frequency, it exponentially decreases with the increase of $f$. The cut-off frequency for model M20.145 is $f_{\text {cut }} \approx 0.04 / m_{0}$. For $f \leq 0.03 / m_{0} \sim 0.8 f_{\text {cut }}, F(f)$ may be approximately fitted by the $3 \mathrm{PN}$ formula for the amplitude [47], and thus, the spectrum may be described, e.g., by the following way

$$
h(f)=h_{3 \mathrm{PN}}(f) e^{-\left(f / f_{\text {cut }}\right)^{\sigma}},
$$

where $h_{3 \mathrm{PN}}$ is the amplitude of gravitational waves derived in the $3 \mathrm{PN}$ theory (see Eq. (79) of Ref. [47]), and $\sigma$ is a constant of order unity. The dotted curve in Fig. 12 (a) denotes the result of the fitting for $f_{\text {cut }}=0.038 / m_{0}$ and $\sigma=2.2$, and we see that the fitting works well. For model M15.145, the fitting also works well and gives $f_{\text {cut }}=0.030 / m_{0}$ and $\sigma=2.2$. Because the NS is tidally disrupted farther outside the ISCO, the value of $f_{\text {cut }}$ is smaller for model M15.145.

The latest high-precision numerical study for the quasiequilibrium states of the BH-NS binaries shows that the mass shedding of the NSs occurs if the following condition is satisfied [7]

$$
\Omega \geq \Omega_{\mathrm{MS}}=C\left(\frac{G M_{\mathrm{NS}}}{R_{\mathrm{NS}}^{3}}\right)^{1 / 2}\left(1+\frac{M_{\mathrm{NS}}}{M_{\mathrm{BH}}}\right)^{1 / 2}
$$

where the value of $C$ is $\approx 0.270$ for the $\Gamma=2$ polytropic EOS. (Note that this is the relation which holds only for the NS of the irrotational velocity field and for the $\mathrm{BH}$ of no spin.) From this relation, we expect that the mass shedding sets in at a frequency $f_{\mathrm{MS}}=\Omega_{\mathrm{MS}} / \pi$. We plot this expected frequency $\left(0.0174,0.0219\right.$, and $\left.0.0265 / m_{0}\right)$ for models M20.145-M40.145 together with the Fourier spectra for models M20.145-M50.145 in Fig. 12 (e).

Figure 12 (a) and (e) show that any special feature is not seen at $f=f_{\mathrm{MS}}$ in the spectrum even for the case that the NS is tidally disrupted before the binary reaches the ISCO, e.g., for model M20.145 (a small bump seen for $f<f_{\mathrm{MS}}$ is due to modulation of the wave amplitude and wave frequency in the numerical data). Also, it is found that $f_{\text {cut }}$ is much larger than $f_{\mathrm{MS}}$, as already pointed out in Ref. [14]. This implies that at $f=f_{\mathrm{MS}}$, the massshedding sets in for the NS, but the NS is not tidally disrupted at such a low frequency. Even for the closer orbits of $f>f_{\mathrm{MS}}$, the NS behaves as a self-gravitating star although it transfers a small amount of mass to the companion $\mathrm{BH}$, and gravitational waves from this BH-NS binary are characterized basically by the inspiral waveforms. However, because the tidal effect becomes more important for the smaller orbital separation, the tidal deformation of the NS is enhanced more with time, and eventually, the tidal disruption occurs. At the tidal disruption, the gravitational wave amplitude should quickly decrease, so it is natural to identify the frequency of the last inspiral wave as $f_{\text {cut }}$ in this case.

Here, we should emphasize that the compactness (or radius) of the $N S s$ is reflected in $f_{\text {cut }}$ not in $f_{\mathrm{MS}}$. In the 
existing idea, one naively assumes that the tidal disruption occurs at $f=f_{\mathrm{MS}}$ and discusses that the radius of the NS (i.e., the EOS of the NS) may be constrained by identifying the values of $f_{\mathrm{MS}}$ (e.g. Ref. [52]). However, our present result indicates that this possibility is unlikely. To constrain the EOS of the NS from gravitational waves detected, we have to theoretically determine $f_{\text {cut }}$ for a variety of the EOSs and masses of the $\mathrm{BH}$ and NS.

For models M20.160 and M30.145 [see Fig. 12 (a) and (b)], the spectrum shape is similar to that for model M20.145, but the cut-off frequency is higher as $f_{\text {cut }} \sim$ $0.06 / m_{0}$ (see below). This cut-off frequency is still lower than the frequency of the fundamental quasinormal mode ( $f_{\mathrm{QNM}}$; see the arrow of Fig. 12 (a) and (b)). This indicates that the tidal disruption occurs at an orbit corresponding to $f=f_{\text {cut }}$. This cut-off frequency, which is higher than that for model M20.145, reflects the fact that the tidal disruption occurs at a closer orbit than that for model M20.145 due to the larger compactness or mass ratio of the system. The cut-off frequency appears to be much higher than the frequency of gravitational waves emitted at the ISCO, which is $f_{\text {lso }} \sim 0.03 / m_{0}$. This implies that gravitational waves of $f_{\text {lso }} \lesssim f \lesssim f_{\text {cut }}$ are not emitted by the inspiral motion of the binary but by a motion in the merger phase. (This is the reason why we do not classify the waveforms for models M20.160 and M30.145 into type I but into type II.) In this case, it is not appropriate to fit the spectrum by Eq. (42) but by a different function (see below).

Another interesting feature found in the spectrum of model M30.145 is that no peak associated with the quasinormal mode appears at $f=f_{\mathrm{QNM}}$ (see arrow in Fig. 12 (b)). The reason is simply that the amplitude of the ringdown waveform is much smaller than that of the merger waveform, as pointed out in Sec. IV C.

For models M40.145, M50.145, and M30.178 (see Fig. 12 (c), (d), and (f)), the gravitational waveforms are type III. In these cases, the spectrum amplitude also steeply decreases above a cut-off frequency, but the feature of the spectrum shape is qualitatively different from those of models M20.145, M30.145, and M20.160, because the gradual decrease of the spectrum amplitude continues approximately up to the frequency of the quasinormal mode (see the arrows in these figures). Namely, $f_{\text {cut }}$ is approximately equal to $f_{\mathrm{QNM}}$, and thus, the cutoff frequency does not indicate that the tidal disruption occurs at an orbit of $f=f_{\text {cut }}$. In other words, the signal of the tidal disruption is absent in these cases.

Another difference is seen in the spectrum shape for a frequency slightly smaller than $f_{\text {cut }}$. For models M20.145, M30.145, and M20.160, a spectral index, $n \equiv-d \ln (f h(f)) / d \ln (f)$, for $f \lesssim f_{\text {cut }}$ monotonically increases with $f$. For models M40.145, M50.145, and M30.178, by contrast, the value of $n$ slightly decreases with $f$, and a "plateau" appears. This spectrum shape is similar to that for the merger of unequal-mass BH$\mathrm{BH}$ binaries [51]. This is reasonable because in this case, tidal effects do not play an important role during the inspiral and merger phases, and the merger process should be qualitatively the same as that in $\mathrm{BH}-\mathrm{BH}$ binaries.

In Fig. 12 (f), we compare the spectrum shape for models M30.145, M30.160, and M30.178, for which the masses of the $\mathrm{BH}$ and NS are identical whereas the compactness of the NS is different. This figure illustrates that for the larger compactness of the NS, the cut-off frequency is higher. Also, the width of the plateau is larger for the more compact models (M30.160 and M30.178). As discussed above, these differences reflect the difference in the evolution process of the late inspiral and merger phases. Because the spectrum shape near $f=f_{\text {cut }}$ is significantly different among three models, observing such high-frequency gravitational waves will play a special role in constraining the compactness (i.e., radius) of the NS.

To systematically identify the cut-off frequency, the fitting of the spectrum with a specific function is useful, as illustrated for model M20.145. The spectra associated with the inspiral phase for models M30.145-M50.145, M20.160, M20.178, M30.160, and M30.178 are also fitted by the $3 \mathrm{PN}$ amplitude, $h_{3 \mathrm{PN}}(f)$, for $f \lesssim f_{\text {lso }} \sim 0.03 / m_{0}$. However, this should not be the case for $f_{\text {lso }} \lesssim f \lesssim f_{\text {cut }}$ because gravitational waves for this high-frequency component are not emitted by the inspiral motion, but by a motion of the material associated with the merger and infalling into the $\mathrm{BH}$. Hence, the spectrum should not be fitted by Eq. (42) but by

$$
h(f)=h_{3 \mathrm{PN}}(f) e^{-\left(f / f_{\mathrm{ins}}\right)^{\sigma}}+h_{\text {merger }}(f),
$$

where $h_{\text {merger }}(f)$ denotes the spectrum of gravitational waves emitted in the merger phase, and $f_{\text {ins }}$ is a frequency of $0.01-0.03 / m_{0}$. In this paper, we fix $\sigma=3.5$ to reduce the total number of free parameters, and choose the following function for $h_{\text {merger }}(f)$,

$$
h_{\text {merger }}(f)=\frac{A m_{0}}{D f} e^{-\left(f / f_{\text {cut }}\right)^{\sigma_{\text {cut }}}}\left[1-e^{-\left(f / f_{\text {ins } 2}\right)^{5}}\right] \text {, }
$$

where $A$ and $\sigma_{\text {cut }}$ are free parameters. We add a free parameter $f_{\text {ins2 }}$ for which the value is close to $f_{\text {ins }}$, because the fitting is achieved in a better manner with it.

The dotted curves in Fig. 12 (b)-(d) denote the results for $\left(f_{\text {ins }} m_{0}, f_{\text {ins } 2} m_{0}, A, f_{\text {cut }} m_{0}, \sigma_{\text {cut }}\right)=(0.014,0.014$, $0.130,0.063,2.90)$ for model M30.145, (0.016, 0.016, $0.103,0.079,4.60)$ for model M40.145, and (0.019, 0.020, 0.090, 0.087, 3.70) for model M50.145. As Fig. 12 shows, the spectrum is well fitted by this simple fitting function.

The fitting for model M50.145 (and M30.178) is not as excellent as those for other models. In these cases, the inclination of the plateau is rather flat in the highfrequency region. This feature is universal for the gravitational waveforms emitted in the merger of $\mathrm{BH}-\mathrm{BH}$ binaries. Thus, in such a case, another fitting function proposed, e.g. in Ref. [51], may be better. The fitting procedure here is, however, still robust in extracting important physical information, as discussed in the following. 


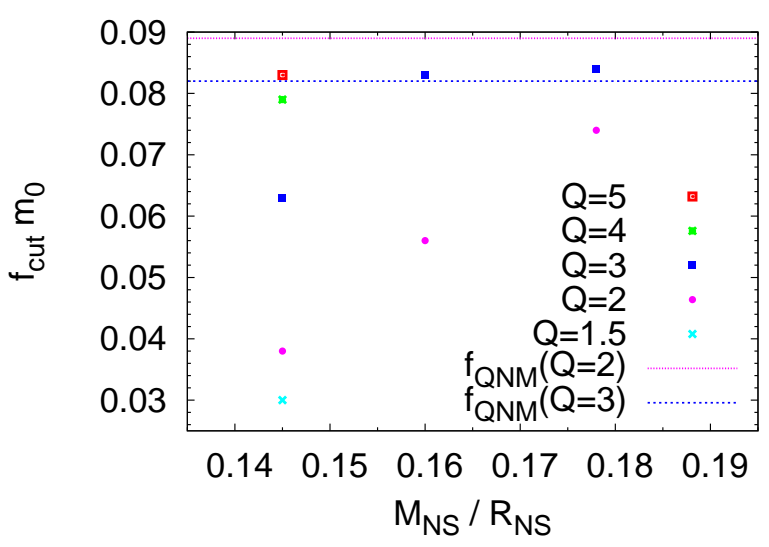

FIG. 13: $f_{\text {cut }}$ as a function of $\mathcal{C}$ for various values of $Q$. The dashed and dotted lines denote the value of $f_{\mathrm{QNM}}$ for $Q=$ 2 and 3 , respectively. For $Q=4$ and $5, f_{\mathrm{QNM}}$ is slightly smaller than that for $Q=3$, and $\approx 0.078 / m_{0}$ and $0.075 / m_{0}$, respectively.

\section{E. Cut-off frequency and determining the compactness of the NS}

In the fitting procedure described in the previous subsections, the most important output is the cut-off frequency, $f_{\text {cut }}$, in which information about the compactness (or radius) of the NS may be reflected. For the case that the NS is swallowed by the $\mathrm{BH}$ with no tidal disruption (e.g., $Q \gtrsim 4$ ), it approximately indicates the frequency of the quasinormal mode of the formed $\mathrm{BH}$, and thus, it will not be possible to extract the compactness of the NSs from the gravitational-wave signal. By contrast, for the case that the NS is tidally disrupted before it is swallowed by the $\mathrm{BH}$ (i.e., for the small values of $Q$ and $\mathcal{C}), f_{\text {cut }}$ is much smaller than $f_{\mathrm{QNM}}$ and indicates a characteristic frequency of gravitational waves emitted at the tidal disruption (see Fig. 13). We find that for models M20.145, M20.160, M20.178, M30.145, and M30.160, $f_{\text {cut }} m_{0} \approx 0.038,0.056,0.070,0.063$, and 0.083 . For $Q=2$, the value of $f_{\text {cut }}$ is smaller than the frequency of the quasinormal mode, $f_{\mathrm{QNM}} \sim 0.09 / m_{0}$ for a wide value of $\mathcal{C}$. This indicates that we will be able to constrain the compactness of the NS if we detect gravitational waves in the merger phase for a binary composed of a low-mass $\mathrm{BH}$ and an NS with $Q \lesssim 2$. For model $\mathrm{M} 30.160, f_{\text {cut }}$ is approximately equal to $f_{\mathrm{QNM}}$, whereas $f_{\text {cut }}<f_{\mathrm{QNM}}$ for mode M30.145. Thus, if the compactness of the NS is fairly small $\mathcal{C}<0.16$, we will have a chance that the detection of gravitational waves from a binary of $Q \approx 3$ constrains the compactness of the NS. By contrast for $Q \geq 4$, gravitational waves are not likely to have robust information about the compactness of the NSs.

Figure 12 (a)-(d) and (f) show that the cut-off frequency is $f=f_{\text {cut }} \sim 1.4-2.2 \mathrm{kHz}$ and the effective amplitude at $f=f_{\text {cut }}$ is universally $\sim 1 \times 10^{-22}$ for hypothetical values $D=100 \mathrm{Mpc}$ and $M_{\mathrm{NS}}=1.35 M_{\odot}$. Even for the optimistic direction of the source with respect to the plane of the detector's arm and of its binary orbital plane, the effective amplitude is at most $\approx 2.5 \times 10^{-22}$. The designed sensitivity of the advanced LIGO is $\leq 3 \times 10^{-22}$ at $f \geq 1 \mathrm{kHz}[53$. This implies that it will not be able to detect gravitational waves during the merger and ringdown phases by the laserinterferometric detectors of standard design for $D \gtrsim 100 \mathrm{Mpc}$. To detect gravitational waves at such high frequency, the detectors composed of special instrument (e.g., resonant-side band extraction), which has a high sensitivity for high-frequency gravitational waves, is necessary. As mentioned above, gravitational waves for $f \lesssim f_{\text {cut }}$ carry important information of the NS radius. It is strongly desired that a detector which is sensitive for the frequency of $1 \mathrm{kHz} \lesssim f \lesssim 3 \mathrm{kHz}$ will be developed in the future.

\section{F. Kick velocity}

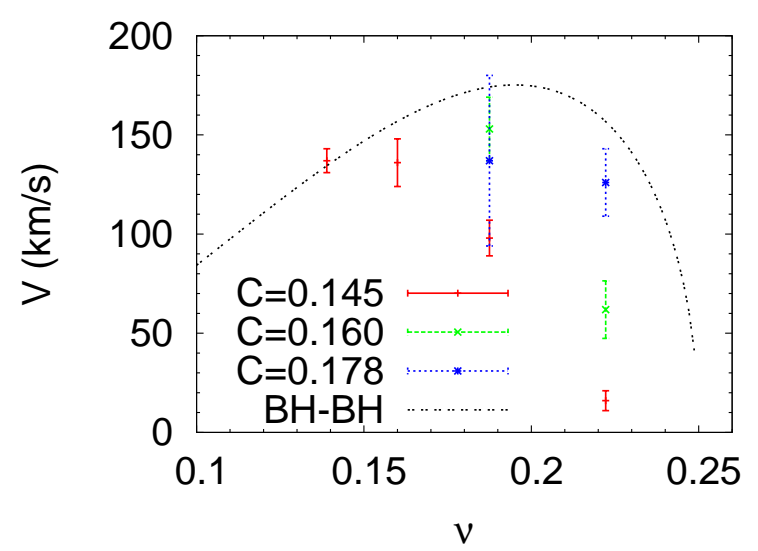

FIG. 14: Kick velocity as a function of $\nu \equiv Q /(1+Q)^{2}$ for different compactness of the NSs. The dot-dotted curve denotes the fitting formula derived in Ref. [40] for the merger of nonspinning $\mathrm{BH}-\mathrm{BH}$ binaries.

Kick velocity induced by anisotropic gravitational radiation is estimated from the total linear momentum carried by gravitational waves. Figure 14 plots the kick velocity as a function of the ratio of reduced mass to total mass for different compactness of the NSs (see also Table IV).

We find that the kick velocity depends strongly on the mass ratio and compactness of the NS. More specifically, it depends strongly on whether the NS is tidally disrupted or not. If the tidal disruption does not occur, the NS simply falls into the companion $\mathrm{BH}$ in a basic picture (although tidal deformation and strong elongation of the NS still occur just before it falls into the $\mathrm{BH}$ ). In such a case, the merger process is similar to that in the merger of nonspinning $\mathrm{BH}-\mathrm{BH}$ binaries, and the kick velocity should agree approximately with the results for those systems. To confirm this fact, we compare our numerical results with the fitting formula derived in Ref. 40] (see dot-dotted curve in Fig. 14). Figure 14 shows that 
our results for models M40.145, M50.145, M30.160, and M30.178 are in a reasonable agreement with the fitting formula of Ref. 40]; the kick velocity is $\sim 100-200 \mathrm{~km} / \mathrm{s}$. This indicates that our numerical results are reliable.

In the case that the NS is tidally disrupted, the kick velocity is significantly suppressed. The primary reason is that the kick is excited most efficiently when two stars merge; more specifically, when the amplitude of gravitational waves becomes maximum. The maximum amplitude of inspiral gravitational waves is higher for the case that two stars have more compact orbits (i.e., the NS is more compact). Also, the amplitude of gravitational waves in the merger phase becomes higher when an NS falls into a companion $\mathrm{BH}$ without tidal deformation. For the case that the tidal disruption happens, close inspiral orbits are prohibited, and as a result, the enhancement of the gravitational-wave amplitude is suppressed as we showed in Sec. IVC, Due to this fact, the kick velocity is smaller for the smaller values of compactness and mass ratio.

\section{SUMMARY}

New general relativistic simulation for the inspiral, merger, and ringdown of BH-NS binaries are performed by a new AMR code SACRA. This paper presents the numerical results of a longterm simulation for a variety of mass ratio and compactness of the NS for the first time. For the simulation, we adopt initial conditions for which the unphysical eccentricity is much smaller than that in our previous study [13, 14], and also the initial orbital separation is much larger. In the present results, the binaries spend in the inspiral phase for 4-7 orbits, and in the last several orbits, the eccentricity is sufficiently suppressed to be $\sim 0.01$ and the orbit becomes approximately quasicircular. This is reflected in successful computation of gravitational waveforms for the inspiral phase which agree well with those predicted by the PN theory. Because the moving-puncture approach is adopted, the merger and subsequent evolution of the $\mathrm{BH}$ with the surrounding material are simulated for a long time until the system relaxes to a quasisteady state. As a result, accurate gravitational waveforms for the late inspiral, merger, and ringdown phases are derived successfully.

By the present self-consistent simulation, we reconfirm the finding in our previous paper [14] that the merger process depends sensitively on the mass ratio, $Q$, and compactness of the NS, $\mathcal{C}$. Only for the case that both $Q$ and $\mathcal{C}$ are sufficiently small, the NS is tidally disrupted by the companion BH. For example, for the chosen EOS in this paper (polytropic EOS with $\Gamma=2$ ), the NS of $\mathcal{C}=0.145$ is tidally disrupted only for $Q \lesssim 3$ before the binary reaches the ISCO. By analyzing the spectrum of gravitational waves, we confirm that even in the case that the NS satisfies the condition for the onset of the mass shedding [see Eq. (43)], the tidal disruption does not occur immediately. Rather, the NS behaves as a self-gravitating star for a while during the mass-shedding phase. Therefore, the tidal disruption occurs for the more restricted case than the mass shedding does. This fact also implies that for determining the condition of tidal disruption, numerical-relativity simulation is the unique approach.

The parameter space for the formation of a long-lived disk surrounding the $\mathrm{BH}$ is even more restricted. For $\mathcal{C}=0.145$, the disk of lifetime $\gg 10 \mathrm{~ms}$ is formed only for $Q \lesssim 2$, and for $\mathcal{C}=0.160$, the disk mass is at most $10^{-3} M_{*}$ even for $Q=2$. The mass of the formed disk is $\gtrsim 0.01-0.02 M_{*}$ for $Q=2-1.5$ even for relatively less compact NSs with $\mathcal{C}=0.145$, and thus, the disk is not very massive, even if it is formed. This conclusion is consistent with the results of Ref. [17].

At present, the precise EOS of high-density nuclear matter is unknown [42]. However, many EOSs predict that the radius of NSs of canonical mass $1.25-1.45 M_{\odot}$ is smaller than $\sim 12 \mathrm{~km}$ (e.g., Ref. [54]). Namely, the compactness of the NS is larger than 0.155. If the EOS is really stiff and the NS is compact as the nuclear theory predicts, the disk will not be formed after the merger between nonspinning $\mathrm{BH}$ and NS, as pointed out by Miller [43]. (However, this will not be the case if the BH has the spin of substantial magnitude.)

We find that gravitational waveforms from BH-NS binaries are roughly classified into the following three types. (i) When the NS is tidally disrupted during the inspiral phase, the gravitational waveform is characterized by the inspiral waveform and subsequent sudden shutdown. In this case, the amplitude of the Fourier spectrum monotonically decreases with the increase of the frequency, and at a cut-off frequency $f_{\text {cut }}$, which is the frequency of gravitational waves when the NS is tidally disrupted (not equal to $f_{\mathrm{MS}}$ ), the spectrum amplitude decreases exponentially [see Eqs. (42) and (44)]. We refer to this waveform as type I in this paper. (ii) In the case that the NS is tidally disrupted but most of the material falls into the companion $\mathrm{BH}$ before the tidal disruption is completed, the gravitational waveform is characterized by the inspiral and merger waveforms. In this case, ringdown gravitational waves are excited in the final phase, but its amplitude is much smaller than those of late inspiral and merger gravitational waves. As a result, the shape of the Fourier spectrum is similar to that of type I but the cut-off frequency does not correspond to the frequency of the last inspiral orbit nor the frequency of the quasinormal mode. We refer to this waveform as type II. (iii) When the NS is not tidally disrupted before it is swallowed by the $\mathrm{BH}$, the gravitational waveform is characterized by the inspiral, merger, and ringdown waveforms. In this case, the amplitude of the Fourier spectrum monotonically decreases with the increase of the frequency in the inspiral phase as in the cases (i) and (ii). However, in the late inspiral and merger phases, the gravitational-wave amplitude increases and, as a result, a plateau appears in the Fourier spectrum of $f \lesssim f_{\text {cut }}$. Then the spectrum amplitude exponentially decreases for 
$f>f_{\text {cut }}$ [see Eq. (44)]. In this case, $f_{\text {cut }}$ is approximately equal to the frequency of the fundamental quasinormal mode of the formed $\mathrm{BH}, f_{\mathrm{QNM}}$, and does not have any information about tidal disruption (and thus compactness of the NS). We refer to this waveform as type III.

We fit the spectrum of gravitational waves with hypothetical functions of a small number of parameters. We find that the fitting works well irrespective of the values of $Q$ and $\mathcal{C}$. By this fitting, we systematically determine the value of the cut-off frequency, $f_{\text {cut }}$ and confirm the following fact: For the case that the NS is not tidally disrupted, the value of $f_{\text {cut }}$ is approximately equal to the value of $f_{\mathrm{QNM}}$, as mentioned above. By contrast, for the case that the tidal disruption occurs, the value of $f_{\text {cut }}$ is smaller than $f_{\mathrm{QNM}}$, and it depends strongly on $Q$ and $\mathcal{C}$. The tidal disruption occurs for a wide range of $\mathcal{C}$ if $Q$ is smaller than $\sim 2$. Thus, if gravitational waves from binaries composed of a low-mass BH and NS at tidal disruption are observed, it may be possible to constrain the compactness of the NS, and as a result, the EOS of the NS.

We also reconfirm that the frequency at the onset of the mass shedding of the NSs is not reflected in the spectrum in an outstanding manner. Namely, the compactness (or radius) of the NSs is reflected in $f_{\text {cut }}$ not in $f_{\mathrm{MS}}$. In the existing idea, one naively assumes that tidal disruption occurs at $f=f_{\mathrm{MS}}$ and discusses that the radius of the NS (i.e., the EOS of the NS) may be constrained by identifying the values of $f_{\mathrm{MS}}$ (e.g. Ref. [52]). However, our present result indicates that this possibility is unlikely. To constrain the EOS of the NS from gravitational waves detected, we have to determine $f_{\text {cut }}$ for a variety of the EOSs and masses of the $\mathrm{BH}$ and NS, that can be done only by numerical-relativity simulation.

Kick velocity induced by asymmetric gravitational wave emission is also computed. When tidal disruption does not occur (e.g., for $Q=5$ ), our result agrees approximately with that derived for the merger of nonspinning $\mathrm{BH}-\mathrm{BH}$ binaries; the kick velocity is $\sim 100-200 \mathrm{~km} / \mathrm{s}$. For the case that the tidal disruption occurs, the kick velocity is significantly suppressed, and it is much smaller than $100 \mathrm{~km} / \mathrm{s}$. This is due to the fact that gravitational wave amplitude in the last inspiral and merger phases is suppressed.

As shown in this paper, gravitational waveforms in the merger and ringdown phases depend strongly on the compactness of the NS and mass ratio of the binary. This results primarily from the fact that the degree of the tidal deformation and condition for the onset of the mass shedding and tidal disruption depend strongly on these two parameters. Gravitational waveforms should also depend on the EOS of the NS and on the spin of the BH, as illustrated, e.g. in Ref. [55]. Thus, in the subsequent work, we will systematically perform the simulation for a variety of the EOSs of the NS and BH spin, to clarify the dependence of the gravitational waveform and its spectrum on these additional parameters.

\section{Acknowledgments}

All the initial conditions for the present numerical simulation are computed using the free library LORENE 28]. We thank members in the Meudon Relativity Group, in particular, Eric Gourgoulhon, for developing LORENE. We also thank Alessandra Buonanno for telling methods for the analysis of numerical gravitational waveforms. The numerical computations were in part performed on NEC-SX8 at Yukawa Institute of Theoretical Physics of Kyoto University. This work was supported by Monbukagakusho Grant No. 19540263 and 20105004.

\section{ERRATUM AND ADDENDUM}

(The original version of this article was submitted on Feb 3, 2009. This erratum is added on Jul 23, 2012.)

In the original version of this article, we reported numerical results obtained by our SACRA code employing a simple $\Gamma$-law equation of state with $\Gamma=2$. During subsequent studies [56, 57], we noticed that we systematically underestimated disk masses in the original work. The reason is that we evolved hydrodynamic variables and estimated disk masses only in a domain of the size $\sim 200^{3} \mathrm{~km}^{3}$, although Einstein's equations were solved in domains of size $\sim 1500^{3}-2000^{3} \mathrm{~km}^{3}$. A small-size domain for hydrodynamics is insufficient for the estimation of the disk mass, if tidal disruption occurs. In particular, for a large NS radius, the tidally disrupted material extends far away from the central region, and the disk mass was significantly underestimated. This problem was found during the writing of [57], and the erratum of [56, 58] were already published.

For this reason, we reperformed simulations for the same models as those of the original version, enlarging the computational domain of hydrodynamics. To estimate disk masses more accurately, in addition, we improved the grid resolutions from $N \leq 36$ to $N=40,50$, and 60 ; with $N=60$, the diameters of NSs are covered by $\approx 100$ grid points, and the diameters of apparent horizons of BHs are by $\approx 20 Q$ grid points. With this improved grid resolution, we also reanalyzed gravitational waves. In addition, the fitting formula for the quasinormal-mode frequency is updated to the latest, more sophisticated one derived in [59].

First, we show the convergence properties of our updated simulations. Figure [15] plots the merger time for $Q=2$ models as a function of $1 / N^{2}\left(\propto \Delta x^{2}\right)$, and indicates that it converges approximately at the second order, which is expected for SACRA. Thus, a reasonable convergence is indicated to be achieved in our new simulations. The convergence properties of other quantities are discussed below.

The corrected quantities for the merger remnant are listed in Table $\mathrm{V}$ for three grid resolutions. This shows that an approximate convergence of the disk mass is 
TABLE V: Corrected numerical results for the remnants: Compare with Table III.

\begin{tabular}{|c|c|c|c|c|c|c|c|c|c|}
\hline Model & $M_{r>r_{\mathrm{AH}}} / M_{*}$ & $\overline{M_{\mathrm{BH}, \mathrm{f}} / M_{0}}$ & $C_{e} / 4 \pi M_{0}$ & $M_{\text {irr }} / M_{0}$ & $\bar{C}_{p} / C_{e}$ & $a_{\mathrm{f} 1}$ & $a_{\mathrm{f} 2}$ & $a_{\mathrm{f} 3}$ & $f_{\text {cut }} m_{0}$ \\
\hline $\mathrm{M} 20.145(N=60)$ & 0.060 & 0.970 & 0.970 & 0.903 & 0.898 & 0.692 & 0.682 & 0.683 & 0.036 \\
\hline $\operatorname{M} 20.145(N=50)$ & 0.065 & 0.969 & 0.968 & 0.902 & 0.899 & 0.693 & 0.679 & 0.680 & 0.037 \\
\hline $\operatorname{M} 20.145(N=40)$ & 0.066 & 0.969 & 0.969 & 0.901 & 0.898 & 0.703 & 0.682 & 0.680 & 0.034 \\
\hline $\mathrm{M} 20.160(N=60)$ & 0.021 & 0.981 & 0.980 & 0.912 & 0.897 & 0.694 & 0.684 & 0.685 & 0.055 \\
\hline $\operatorname{M} 20.160(N=50)$ & 0.025 & 0.980 & 0.980 & 0.911 & 0.897 & 0.697 & 0.684 & 0.685 & 0.054 \\
\hline $\operatorname{M} 20.160(N=40)$ & 0.028 & 0.980 & 0.979 & 0.910 & 0.897 & 0.704 & 0.684 & 0.685 & 0.052 \\
\hline $\operatorname{M} 20.178(N=60)$ & 0.0021 & 0.983 & 0.982 & 0.917 & 0.903 & 0.680 & 0.667 & 0.667 & 0.078 \\
\hline $\operatorname{M} 20.178(N=50)$ & 0.0027 & 0.984 & 0.982 & 0.917 & 0.905 & 0.688 & 0.666 & 0.662 & 0.075 \\
\hline $\operatorname{M} 20.178(N=40)$ & 0.0067 & 0.984 & 0.982 & 0.916 & 0.902 & 0.701 & 0.670 & 0.662 & 0.069 \\
\hline $\operatorname{M} 30.145(N=60)$ & 0.044 & 0.978 & 0.978 & 0.936 & 0.936 & 0.566 & 0.556 & 0.557 & 0.065 \\
\hline M30.145 $(N=50)$ & 0.047 & 0.978 & 0.978 & 0.935 & 0.936 & 0.585 & 0.557 & 0.558 & 0.063 \\
\hline $\operatorname{M30.145}(N=40)$ & 0.055 & 0.977 & 0.976 & 0.934 & 0.936 & 0.573 & 0.556 & 0.558 & 0.056 \\
\hline $\operatorname{M} 30.160(N=60)$ & 0.014 & 0.982 & 0.982 & 0.940 & 0.937 & 0.565 & 0.554 & 0.555 & 0.080 \\
\hline M30.160 $(N=50)$ & 0.017 & 0.982 & 0.981 & 0.939 & 0.937 & 0.569 & 0.554 & 0.555 & 0.079 \\
\hline $\operatorname{M30.160}(N=40)$ & 0.023 & 0.982 & 0.981 & 0.939 & 0.936 & 0.578 & 0.556 & 0.555 & 0.075 \\
\hline M30.178 $(N=60)$ & 0.0011 & 0.983 & 0.982 & 0.941 & 0.938 & 0.561 & 0.547 & 0.548 & 0.086 \\
\hline M30.178 $(N=50)$ & 0.0020 & 0.983 & 0.982 & 0.941 & 0.938 & 0.568 & 0.549 & 0.550 & 0.086 \\
\hline $\operatorname{M30.178}(N=40)$ & 0.0039 & 0.984 & 0.983 & 0.941 & 0.937 & 0.580 & 0.551 & 0.550 & 0.083 \\
\hline $\mathrm{M} 40.145(N=60)$ & 0.016 & 0.986 & 0.985 & 0.955 & 0.955 & 0.485 & 0.475 & 0.477 & 0.080 \\
\hline $\mathrm{M} 40.145(N=50)$ & 0.018 & 0.985 & 0.985 & 0.955 & 0.955 & 0.486 & 0.475 & 0.476 & 0.079 \\
\hline $\mathrm{M} 40.145(N=40)$ & 0.026 & 0.985 & 0.984 & 0.954 & 0.955 & 0.492 & 0.474 & 0.476 & 0.076 \\
\hline $\mathrm{M} 50.145(N=60)$ & $4 \times 10^{-4}$ & 0.990 & 0.989 & 0.966 & 0.966 & 0.426 & 0.417 & 0.418 & 0.082 \\
\hline $\operatorname{M50.145}(N=50)$ & $7 \times 10^{-4}$ & 0.990 & 0.989 & 0.967 & 0.965 & 0.431 & 0.418 & 0.420 & 0.082 \\
\hline $\operatorname{M50.145}(N=40)$ & 0.0022 & 0.991 & 0.990 & 0.966 & 0.965 & 0.435 & 0.419 & 0.420 & 0.080 \\
\hline
\end{tabular}

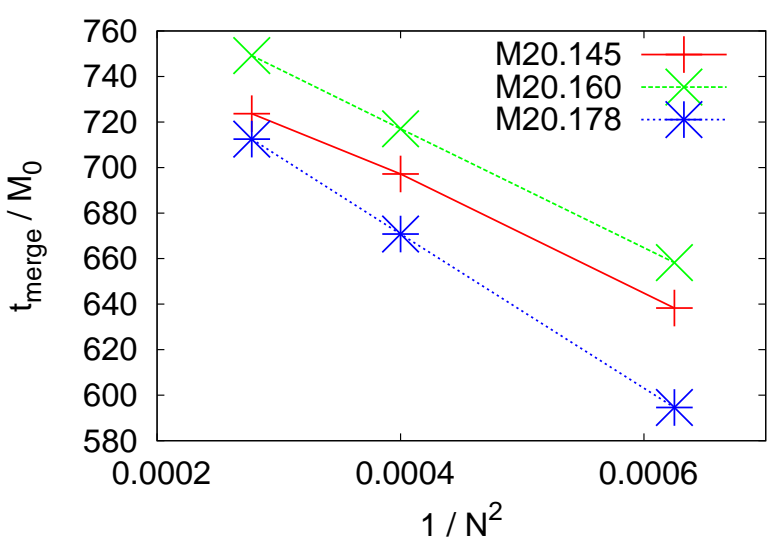

FIG. 15: The merger time normalized by the Arnowitt-DeserMisner mass of the initial configuration for models with $Q=2$ as a function of $1 / N^{2}$. The left, middle, and right points correspond to the results of $N=60,50$, and 40 , respectively.

achieved for cases $M_{r>r_{\mathrm{AH}}} \gtrsim 0.01 M_{*}$, and the convergence appears to be not so good when $M_{r>r_{\mathrm{AH}}} \lesssim 0.01 M_{*}$. This is simply because such a small disk mass is supplied from a lower-density region near the surface of NSs for which an accurate computation is forbidden in a poor-

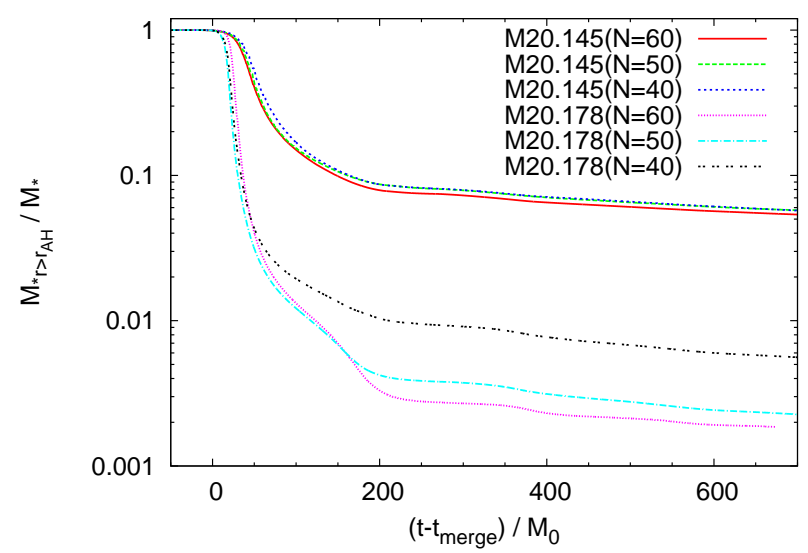

FIG. 16: Evolution of the rest mass of the material located outside the apparent horizon for different grid resolutions of models M20.145 and M20.178.

resolution run; i.e., the steep density gradients near the surface cannot be accurately resolved, leading to a slight spurious expansion of the surface which spuriously increases the resulting disk mass. We estimate that the disk mass is overestimated even in the run with $N=60$ by a factor of $2-3$, when the resulting disk mass is of order $10^{-3} M_{*}$ or less. Figure 16 plots the evolution of 
$M_{r>r_{\mathrm{AH}}}$ for three grid resolutions. This indeed indicates that the convergence is good when $M_{r>r_{\mathrm{AH}}} \gtrsim 0.01 M_{*}$ but not as good when $M_{r>r_{\mathrm{AH}}} \sim 10^{-3} M_{*}$. Although the remnant disk mass may be overestimated by $10^{-3} M_{*}-$ $10^{-2} M_{*}$, this error is acceptable for the problem of disk formation with $\sim 0.1 M_{*}$. We also note that our results of the disk mass for models M30.145 are in approximate agreement with those of numerical-relativity simulations performed by other two groups $[60,61]$.

Table $\mathrm{V}$ shows that corrected quantities of the remnant $\mathrm{BH}$ are in the convergent regime within $O(0.1) \%$ errors except for $a_{\mathrm{f} 1}$, indicating a reasonable convergence. The reason for the poorer convergence of $a_{\mathrm{f} 1}$ is due to its dependence on the mass and angular momentum of the remnant disk. However, we find that the disagreement between $a_{\mathrm{f} 1}$ and other two, $a_{\mathrm{f} 2}$ and $a_{\mathrm{f} 3}$, becomes smaller as the grid resolution is improved, and the error is $\lesssim$ 0.015 for $N=60$. We note that $M_{\mathrm{BH}, \mathrm{f}}$ and $C_{e} / 4 \pi$ always agree approximately with each other irrespective of $N$.

The corrected remnant disk mass for $N=60$ is significantly larger than that in our previous simulations. The reason for the mistake in the original version is due to the fact that we did not follow the motion of the material which is ejected by tidal disruption and escapes outside the central domain of the size $\sim 200^{3} \mathrm{~km}^{3}$, even when they are bound and supposed to eventually return to the neighborhood of the BH. The new SACRA can correctly estimate the mass of the returned material. Snapshots of the rest-mass density profiles for model M30.145 are displayed in Fig. 17, which shows that the hydrodynamics processes are solved appropriately in the far region. Figure 18 plots the time evolution of $M_{r>r_{\mathrm{AH}}}$ for all the models with $N=60$. It is found that the remnant disk mass after $\approx 10 \mathrm{~ms}$ of the merger exceeds $0.01 M_{\odot}$ for a wider range of binary parameters (for a hypothetical NS mass $\left.1.35 M_{\odot}\right)$. However, the remnant disk mass is still small for a large mass ratio, $Q=5$ or for a compact NS, $\mathcal{C}=0.178$ for which the tidal effect plays a minor role in the merger process.

By contrast, quantities associated with the remnant $\mathrm{BH}$ do not differ much from those obtained in the original version. This fact suggests that the disrupted material does not affect the remnant $\mathrm{BH}$ strongly. This is reasonable, because the mass of the remnant $\mathrm{BH}$ is always much larger than that of the remnant disk.

The gravitational waveforms for selected models are plotted in Fig. 19. As often found in our series of papers [56, 57], the waveform agrees well with the Taylor-T4 formula except for the first one orbit and for the final inspiral orbit just before the merger. For completeness, corrected results for the radiated energy and angular momentum are also listed in Table VI.

The gravitational-wave spectra for selected models are shown in the left panel of Fig. 20 together with the spectra computed by the Taylor-T4 formula and of the phenomenological waveform proposed in 62] following the idea of 61]. We also recomputed the cutoff frequency, $f_{\text {cut }}$, for each model, and the results are listed in Table. [V. We reconfirm the correlation between $\mathcal{C}$ and $f_{\text {cut }} m_{0}$ for the fixed $Q$ (the cutoff frequency converges approximately for $N=60$ and 50). The right panel of Fig. 20] shows the spectrum for selected models with different grid resolutions, and indicates that the spectrum is in the convergent regime for $N=60$ and 50 .

Finally, we would like to apologize to readers of the original version for any inconvenience caused by our negligence.
[1] S. J. Waldman et al., Class. Quantum Grav. 23, S653 (2006). See also http://www.ligo.caltech.edu/

[2] F. Acernese et al., Class. Qauntum Grav. 23, S635 (2006). See also http://www.virgo.infn.it/

[3] R. Voss and T. M. Taulis, Mon. Not. R. Astro. Soc. 342, 1169 (2003); K. Belczynski, T. Bulik, and B. Rudak, Astrophys. J. 571, 394 (2002); V. Kalogera et al., Astrophys. J. 601, L179 (2004); V. Kalogera et al., Phys. Rep. 442, 75 (2007).

[4] K. Belczynski, R. E. Taam, V. Kalogera, F. A. Rasio, and T. Bulik, Astrophys. J. 662, 504 (2007): This paper suggests that the merger rate may be much lower than that predicted by the earlier works [3].

[5] http://www.ligo.caltech.edu/advLIGO/

[6] K. Taniguchi, T. W. Baumgarte, J. A. Faber, and S. L. Shapiro, Phys. Rev. D 75, 084005 (2007).

[7] K. Taniguchi, T. W. Baumgarte, J. A. Faber, and S. L. Shapiro, Phys. Rev. D 77, 044003 (2008).

[8] F. Foucart, L. E. Kidder, H. P. Pfeiffer, and S. A. Teukolsky, Phys. Rev. D 77, 124051 (2008).

[9] C. L. Fryer, S. E. Woosley, M. Herant, and M. B. Davies, Astrophys. J. 520, 650 (1999).
[10] E. Barger, in AIP Conf. Proc. 836, 33 (2006).

[11] R. Popham, S. E. Woosley, and C. Fryer, Astrophys. J. 518 (1999), 356; R. Narayan, T. Piran, and P. Kumar, Astrophys. J. 557 (2001), 949; T. DiMatteo, R. Perna, and R. Narayan, Astrophys. J. 579 (2002), 706; K. Kohri and S. Mineshige, Astrophys. J. 577 (2002), 311; A. Janiuk, R. Perna, T. DiMatteo, and B. Czerny, Mon. Not. R. Astron. Soc. 355 (2004), 950; W.-X. Chen and A. M. Beloborodov, Astrophys. J. 657, 383 (2007).

[12] S. Setiawan, M. Ruffert, and H.-Th. Janka, Mon. Not. R. Astron. Soc. 352, 753 (2004); Astron. Astrophys. 458, 553 (2006); W. H. Lee, E. Ramirez-Ruiz, and D. Page, Astrophys. J. 632, 421 (2005); M. Shibata, Y.I. Sekiguchi, and R. Takahashi, Prog. Theor. Phys. 118, 257 (2007).

[13] M. Shibata and K. Uryū, Phys. Rev. D 74, 121503(R) (2006); Class. Quantum Grav. 24, S125 (2007).

[14] M. Shibata and K. Taniguchi, Phys. Rev. D 77, 084015 (2008).

[15] Z. B. Etienne, J. A. Faber, Y.T. Liu, S. L. Shapiro, K. Taniguchi, and T. W. Baumgarte, Phys. Rev. D 77, 084002 (2008). 

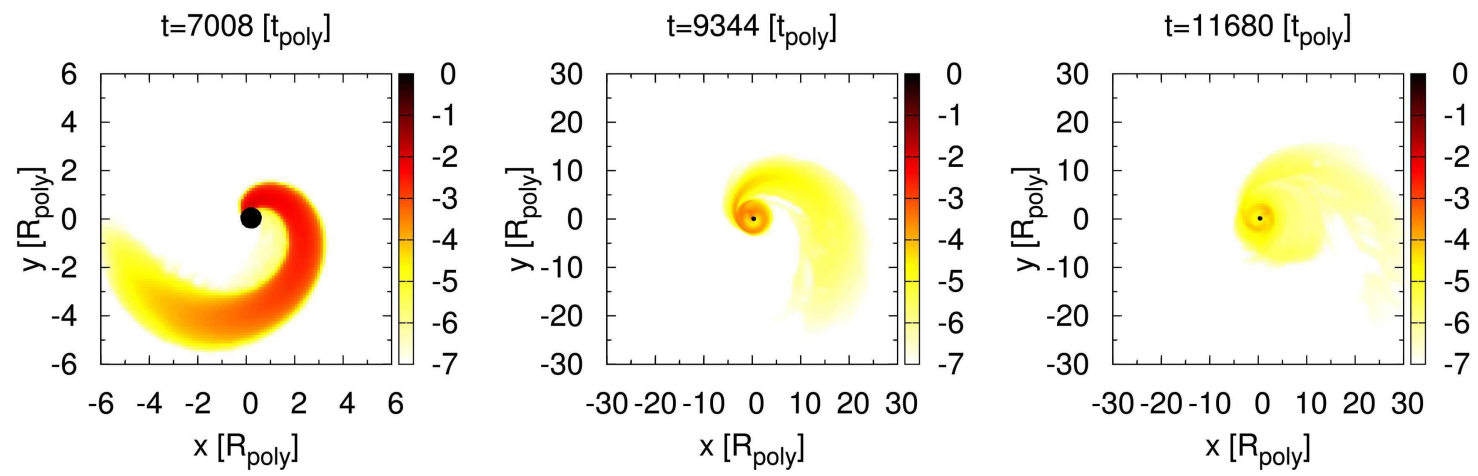

FIG. 17: Evolution of the rest-mass density profile in the logarithmic scale and the location of apparent horizons (black filled circles) for model M30.145 $(\mathrm{N}=60)$. All the quantities are shown in the polytropic unit, in which the unit length is $\approx 14 \mathrm{~km}$ for a hypothetical NS mass $M_{\mathrm{NS}}=1.35 M_{\odot}$.
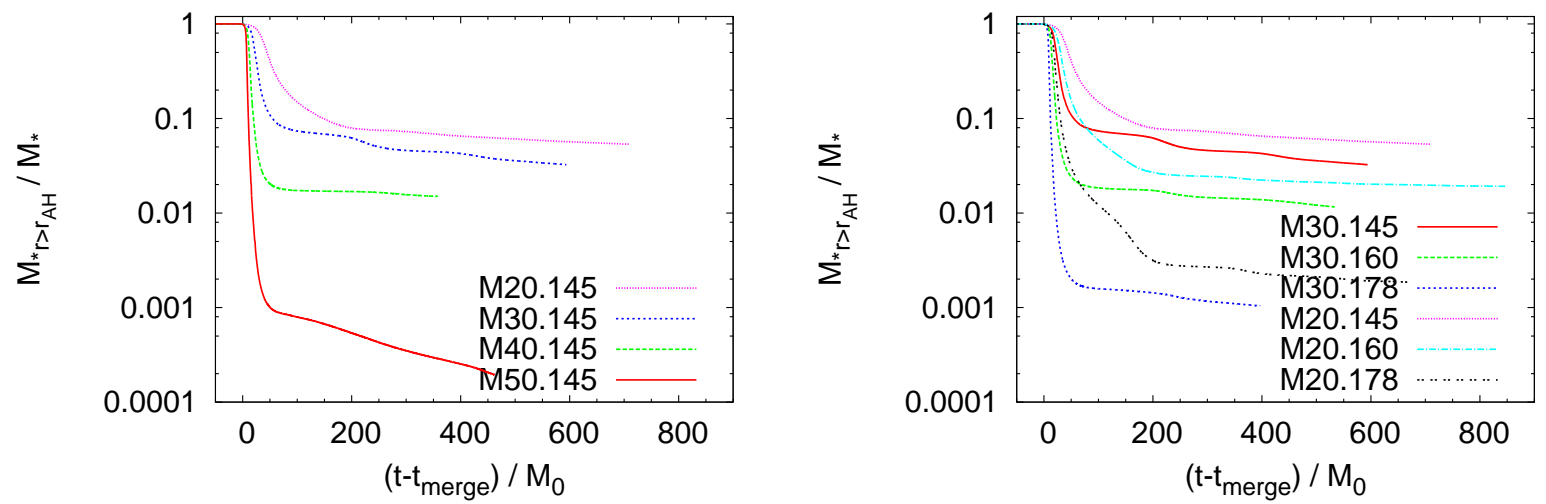

FIG. 18: Left: Evolution of the rest mass of the material located outside apparent horizons for models M20.145, M30.145, M40.145, and M50.145 in $N=60$ runs. Right: The same as the left panel, but for models M30.145, M30.160, M30.178, M20.145, M20.160, M20.178 in $N=60$ runs. For a hypothetical NS mass $M_{\mathrm{NS}}=1.35 M_{\odot}, 100 M_{0} \approx 2.00(Q+1) / 3$ ms.

[16] T. Yamamoto, M. Shibata, and K. Taniguchi, Phys. Rev. D 78, 064054 (2008).

[17] M. D. Duez, F. Foucart, L. E. Kidder, H. P. Pfeiffer, M. A. Scheel, and S. A. Teukolsky, Phys. Rev. D 78, 104015 (2008).

[18] S. Brandt and B. Brügmann, Phys. Rev. Lett. 78, 3606 (1997).

[19] M. Campanelli, C. O. Lousto, P. Marronetti, and Y. Zlochower, Phys. Rev. Lett. 96, 111101 (2006); J. G. Baker, J. Centrella, D.-I. Choi, M. Koppitz, and J. van Meter, Phys. Rev. Lett. 96, 111102 (2006).

[20] P. Grandclément, Phys. Rev. D 74, 124002 (2006) [Erratum ibid. 75, 129903 (2007)].

[21] K. Taniguchi, T. W. Baumgarte, J. A. Faber, and S. L. Shapiro, Phys. Rev. D 72, 044008 (2005).

[22] G. B. Cook, Living Reviews in Relativity, Vol. 3, lrr2000-5 (2000).

[23] K. Kyutoku, M. Shibata, and K. Taniguchi, in preparation.

[24] C. S. Kochanek, Astrophys. J. 398, 234 (1992); L. Bildsten and C. Cutler, ibid 400, 175 (1992).

[25] M. Shibata, Phys. Rev. D 58, 024012 (1998); S. A. Teukolsky, Astrophys. J. 504, 442 (1998).

[26] B. Brügmann, J. A. Gonzalez, M. Hannam, S. Husa, U. Sperhake, and W. Tichy, Phys. Rev. D 77, 024027 (2008).

[27] E. Gourgoulhon, P. Grandclement, and S. Bonazzola,
Phys. Rev. D 65, 044020 (2002); P. Grandclement, E. Gourgoulhon, and S. Bonazzola, Phys. Rev. D 65, 044021 (2002). G. B. Cook, Phys. Rev. D 65, 084003 (2002).

[28] LORENE website: http://www.lorene.obspm.fr/.

[29] L. Blanchet, Living Rev. Relativ. Vol. 9, lrr-2006-4 (2006).

[30] E. Berti, S. Iyer, and C. M. Will, Phys. Rev. D 77, 024019.

[31] P. C. Peters and J. Mathews, Phys. Rev. 131, 435 (1963).

[32] S. Husa, M. Hannam, J. A. González, U. Sperhake, and B. Brügmann, Phys. Rev. D 77, 044037 (2008).

[33] I. H. Stairs, Science 304, 547 (2004).

[34] M. Shibata and T. Nakamura, Phys. Rev. D 52, 5428 (1995); T. W. Baumgarte and S. L. Shapiro, Phys. Rev. D 59, 024007 (1998).

[35] P. Marronetti, W. Tichy, B. Brügmann, J. A. González, and U. Sperhake, Phys. Rev. D 77, 064010 (2008).

[36] M. Hannam, S. Husa, D. Pollney, B. Brügmann, and N. ÓMurchadha, Phys. Rev. Lett. 99, 241102 (2007).

[37] A. Kurganov and E. Tadmor, J. Comput. Phys. 160, 241 (2000).

[38] M. Shibata, Phys. Rev. D 67, 024033 (2003).

[39] M. Shibata, Phys. Rev. D 55, 2002 (1997): M. Shibata and K. Uryū, Phys. Rev. D 62, 087501 (2000).

[40] J. A. Gonzalez, U. Sperhake, B. Brügmann, M. Hannam, 

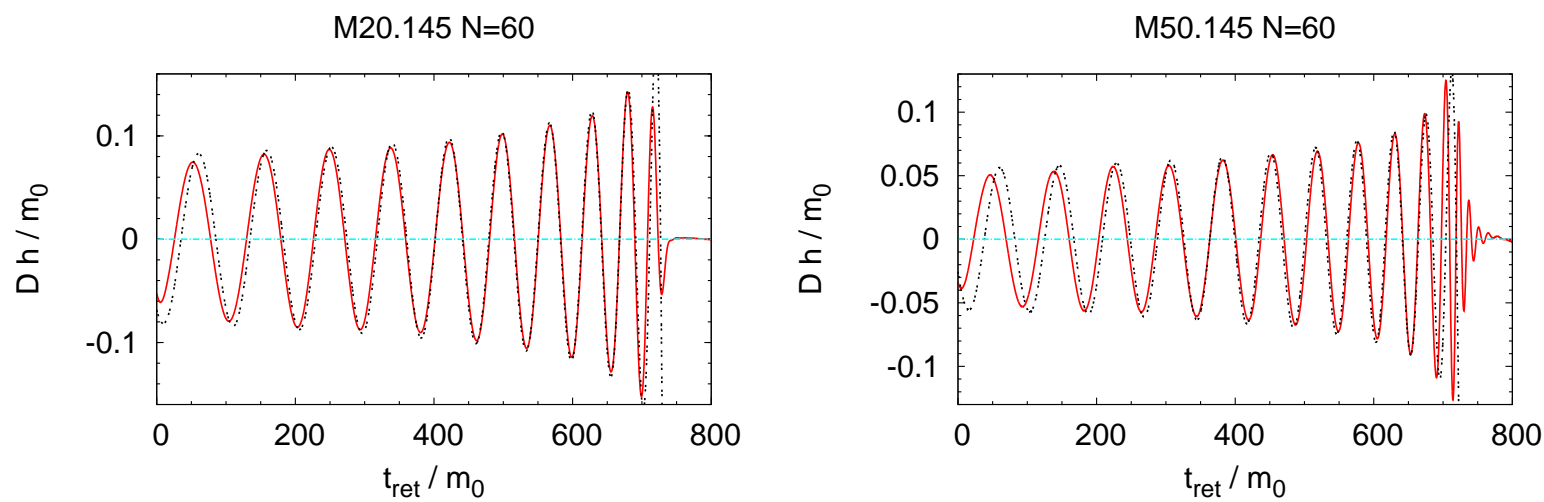

FIG. 19: Gravitational waveforms observed along the $z$ axis (solid curve) for models M20.145 (left) and M50.145 (right). $t_{\text {ret }}$ denotes the retarded time and $m_{0}$ is the total mass. The solid curves denote the results obtained in $N=60$ runs and the dot-dotted curves denote the waveforms derived by the Taylor-T4 formula.

TABLE VI: Corrected numerical results for gravitational waves: Compare with Table IV.

\begin{tabular}{ccccccccc}
\hline \hline Model & $\Delta E / M_{0}(\%)$ & $\Delta J / J_{0}(\%)$ & $(2,2)$ & $(3,3)$ & $(4,4)$ & $(2,1)$ & $f_{\mathrm{QNM}} M_{\mathrm{BH}}$ & $V_{\text {kick }}(\mathrm{km} / \mathrm{s})$ \\
\hline M20.145 $(N=60)$ & 0.80 & 17 & 0.78 & 0.014 & 0.004 & 0.001 & 0.084 & 25 \\
M20.160 $(N=60)$ & 1.13 & 20 & 1.10 & 0.024 & 0.007 & 0.003 & 0.084 & 43 \\
M20.178 $(N=60)$ & 1.62 & 23 & 1.56 & 0.037 & 0.010 & 0.008 & 0.083 & 80 \\
M30.145 $(N=60)$ & 0.99 & 18 & 0.93 & 0.041 & 0.009 & 0.003 & 0.077 & 20 \\
M30.160 $(N=60)$ & 1.37 & 22 & 1.28 & 0.068 & 0.012 & 0.007 & 0.077 & 15 \\
M30.178 $(N=60)$ & 1.70 & 24 & 1.56 & 0.11 & 0.017 & 0.010 & 0.077 & 40 \\
M40.145 $(N=60)$ & 1.09 & 20 & 0.97 & 0.084 & 0.018 & 0.016 & 0.073 & 92 \\
M50.145 $(N=60)$ & 1.01 & 20 & 0.86 & 0.11 & 0.022 & 0.012 & 0.071 & 84 \\
\hline \hline
\end{tabular}

S. Husa, Phys. Rev. Lett. 98, 091101 (2007).

[41] J. D. Schnittman et al., arXiv:0707.0301.

[42] J. M. Lattimer and M. Prakash, Science 304, 536 (2004).

[43] M. C. Miller, Astrophys. J. 626, L41 (2005).

[44] M. Shibata, Phys. Rev. D 76, 064035 (2007).

[45] A. Buonanno, G. B. Cook, and F. Pretorius, Phys. Rev. D 75, 124018 (2007).

[46] M. Boyle, D. A. Brown, L. E. Kidder, A. H. Mroue, H. P. Pfeiffer, M. A. Scheel, G. B. Cook, and S. A. Teukolsky, Phys. Rev. D 76, 124038 (2007); M. Boyle, et al., arXiv:0804.4184.

[47] L. E. Kidder, Phys. Rev. D 77, 044016 (2008).

[48] T. Nakamura and M. Sasaki, Phys. Lett. 106B, 69 (1981).

[49] E. W. Leaver, Proc. R. Soc. Lond. A 402, 285 (1985); F. Echeverria, Phys. Rev. D 40, 3194 (1989).

[50] C. Cutler and E. E. Flanagan, Phys. Rev. D 49, 2658 (1994).

[51] P. Ajith et al., Phys. Rev. D 77, 104017 (2008).

[52] M. Vallisneri, Phys. Rev. Lett. 84, 3519 (2000).

[53] K. S. Thorne, in Compact Stars in binaries: the proceeding of IAU symposium No. 165 (Kluwer Academic Publishers, Dordrecht, 1996), pp. 153.
[54] A. Akmal, V. R. Pandharipande, and D. G. Ravenhall, Phys. Rev. C 58, 1804 (1998); F. Douchin and P. Haensel, Astron. Astrophys. 380, 151 (2001).

[55] M. Ishii, M. Shibata, and Y. Mino, Phys. Rev. D 71, 044017 (2005).

[56] K. Kyutoku, M. Shibata, and K. Taniguchi, Phys. Rev. D 82, 044049 (2010)

[57] K. Kyutoku, H. Okawa, M. Shibata, and K. Taniguchi, Phys. Rev. D 84, 064018 (2011)

[58] K. Kyutoku, M. Shibata, and K. Taniguchi, Phys. Rev. D 84, 049902(E) (2011)

[59] E. Berti, V. Cardoso, and A. O. Starinets, Class. Quant. Grav. 26, 163001 (2009)

[60] M. D. Duez, F. Foucart, L. E. Kidder, C. D. Ott, and S. A. Teukolsky, Class. Quant. Grav. 27, 114106 (2010)

[61] Z. B. Etienne, Y. T. Liu, S. L. Shapiro, and T. W. Baumgarte, Phys. Rev. D 79, 044024 (2009)

[62] P. Ajith, S. Babak, Y. Chen, M. Hewitson, B. Krishnan, A. M. Sintes, J. T. Whelan, B. Brügmann, P. Diener, N. Dorband, J. Gonzalez, M. Hannam, S. Husa, D. Pollney, L. Rezzolla, L. Santamaría, U. Sperhake, and J. Thornburg, Phys. Rev. D 77, 104017 (2008) 

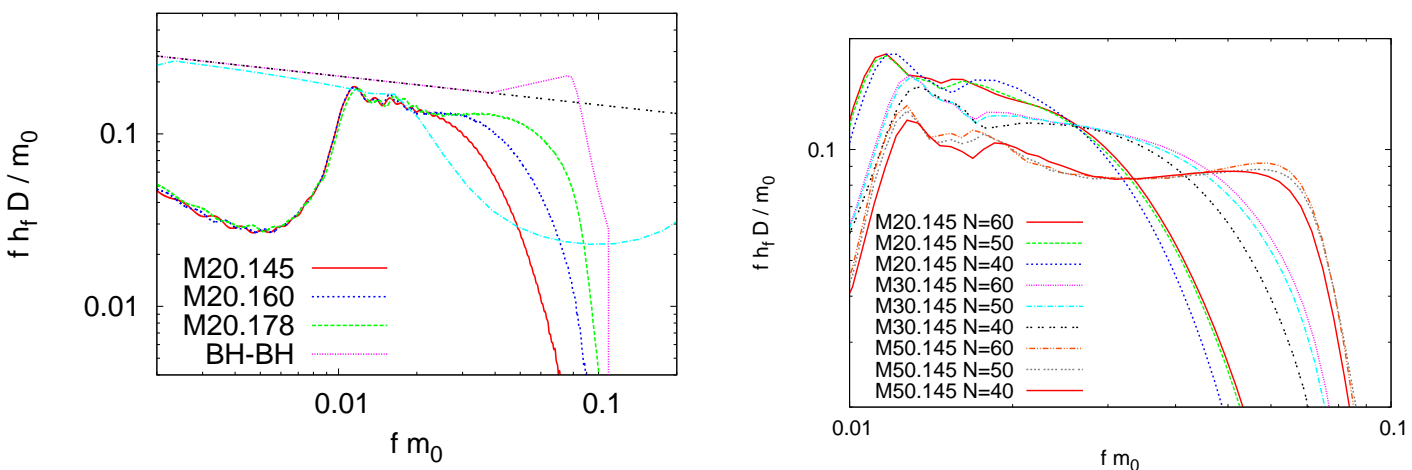

FIG. 20: Left: The spectrum of gravitational waves $f h(f) D / m_{0}$ for models M20.145, M20.160, and M20.178 with $N=60$. The dashed and long-dashed curves denote spectra computed in the quadrupole and Taylor-T4 formulae, respectively. The curve labeled "BH-BH" is the spectrum of the phenomenological waveform for binary black holes proposed in [62]. Right: The spectra for models M20.145, M30.145, and M50.145 with different grid resolutions. We do not match numerical data to the Taylor-T4 waveform unlike in our original version. 
This figure "allfig4.jpg" is available in "jpg" format from: http://arxiv.org/ps/0902.0416v2 
This figure "allfig5.jpg" is available in "jpg" format from: http://arxiv.org/ps/0902.0416v2 
This figure "allfig6.jpg" is available in "jpg" format from: http://arxiv.org/ps/0902.0416v2 